\title{
On-line monitoring of radiotherapy beams: Experimental results with proton beams
}

\author{
D. W. Litzenberg, ${ }^{\text {a) }}$ D. A. Roberts, M. Y. Lee, K. Pham, A. M. Vander Molen, \\ R. Ronningen, and F. D. Becchetti \\ The University of Michigan Medical Center, Department of Radiation Oncology, \\ UH-Rm. B2C432B Box 0010, 1500 E. Medical Center Drive, Ann Arbor, Michigan 48109
}

(Received 25 June 1998; accepted for publication 19 March 1999)

\begin{abstract}
Proton radiotherapy is a powerful tool in the local control of cancer. The advantages of proton radiotherapy over gamma-ray therapy arise from the phenomenon known as the Bragg peak. This phenomenon enables large doses to be delivered to well-defined volumes while sparing surrounding healthy tissue. To fully realize the potential of this technique the location of the high-dose volume must be controlled very accurately. An imaging system was designed and tested to monitor the positron-emitting activity created by the beam as a means of verifying the beam's range, monitoring dose, and determining tissue composition. The prototype imaging system consists of 12 pairs of cylindrical BGO detectors shielded in lead. Each crystal was $1.9 \mathrm{~cm}$ in diameter, $5.0 \mathrm{~cm}$ long, and separated by $0.5 \mathrm{~cm}$ from other detectors in the row. These are arranged in two rows, $60 \mathrm{~cm}$ apart, with the proton beam and tissue phantoms half-way between and parallel to the detector rows. Experiments were conducted with $150 \mathrm{MeV}$ continuous and macro-pulsed proton beams which had beam currents ranging from $0.14 \mathrm{nA}$ to $1.75 \mathrm{nA}$. The production and decay of short-lived isotopes, ${ }^{15} \mathrm{O}$ and ${ }^{14} \mathrm{O}$, was studied using $1 \mathrm{~min}$ irradiations with a continuous beam. These isotopes provide a significant signal on short time scales, making on-line imaging possible. Macro-pulsed beams, having a period of $10 \mathrm{~s}$, were used to study on-line imaging and the production and decay of long-lived isotopes, ${ }^{13} \mathrm{~N},{ }^{11} \mathrm{C}$, and ${ }^{18} \mathrm{~F}$. Decay data were acquired and on-line images were obtained between beam pulses and indicate that range verification is possible, for a $150 \mathrm{MeV}$ beam, after one beam pulse, to within the $1.2 \mathrm{~cm}$ resolution limit of the imaging system. The dose delivered to the patient may also be monitored by observing the increase in the number of coincidence events detected between successive beam pulses. Over $80 \%$ of the initial positron-emitting activity is from ${ }^{15} \mathrm{O}$ while the remainder is primarily ${ }^{11} \mathrm{C},{ }^{13} \mathrm{~N},{ }^{14} \mathrm{O}$ with traces of ${ }^{18} \mathrm{~F}$, and ${ }^{10} \mathrm{C}$. Radioisotopic imaging may also be performed along the beam path by fitting decay data collected after the treatment is complete. Using this technique, it is shown that variations in elemental composition in inhomogenous treatment volumes may be identified and used to locate anatomic landmarks. Radioisotopic imaging also reveals that ${ }^{14} \mathrm{O}$ is created well beyond the Bragg peak, apparently by secondary neutrons. (C) 1999 American Association of Physicists in Medicine.
\end{abstract}

[S0094-2405(99)02206-3]

Key words: proton radiotherapy, nuclear activation, range verification, isotopic imaging

\section{INTRODUCTION}

Proton and light-ion radiotherapy are increasingly important tools for treating tumors due to the sharp rise and fall of dose at the end of the ions range. This advantage over other radiotherapy modalities is limited in many situations by the inability to directly monitor the dose delivered to the tumor volume. The depth of the maximum dose is dependent on the beam energy, tissue composition, multiple Coulomb scattering, ${ }^{1}$ and in particular the inhomogeneities along the beam path. ${ }^{2-4}$ This makes it very difficult to determine the precise range of the treatment beam. The introduction of $\mathrm{X}$-ray CT in treatment planning greatly improved dose localization. However, it has been shown that in the presence of bone or air gaps along the beam path, CT range estimates may be in error by more than $10 \% .^{5,6}$ Thus it is desirable to develop on-line, in vivo techniques for imaging induced activity, to help ensure that the lethal dose is localized within the prescribed tumor volume.
Tissue activation was first discussed in detail by Tobias in $1949^{7}$ and was first reported in gamma-ray experiments by Mayneord et al. later that year. ${ }^{8}$ Since then the radioactivity induced in tissues by photon, ${ }^{8-10}$ proton, ${ }^{11,12}$ neutron, ${ }^{13}$ pion, ${ }^{14-16}$ and heavy-ion radiotherapy beams ${ }^{17-19}$ has been investigated. This induced activity has been studied as a source of patient dose, ${ }^{11,20,21}$ as a means of determining elemental tissue composition, ${ }^{10,11,13}$ and for determining dose distributions. ${ }^{14,15,17,22-31}$ In vivo dose localization by imaging positron-emitting nuclei is considered essential for light-ion, and radioactive-ion beam therapy. ${ }^{30}$ Investigations of PET as a monitor for proton radiotherapy beams generally indicate that it is capable of measuring useful dosimetric, compositional, and range information. ${ }^{11,12,22,25-29,31}$ However, many of these experiments were conducted off-line by detection systems in the vicinity of the treatment beam requiring that the phantoms or subjects under study be moved. The resulting images predominantly show activity from radioisotopes 
whose half-life is comparable to or longer than the transportation and setup time. While these studies lend valuable insight, the images obtained are mainly of ${ }^{11} \mathrm{C}$. Because ${ }^{11} \mathrm{C}$ is produced in relatively small amounts and has a 20 min halflife, the images obtained tend to have poor statistics and require an uncomfortably long imaging time for the patient. In contrast, ${ }^{15} \mathrm{O}$ is the most abundant positron-emitting isotope produced during proton radiotherapy due to the abundance of oxygen in the body, its short 2 min half-life, and the relatively large cross section of the ${ }^{16} \mathrm{O}(p, p n){ }^{15} \mathrm{O}$ reaction. ${ }^{32}$ Therefore, statistically significant images of ${ }^{15} \mathrm{O}$ may be obtained in seconds or minutes. Such images are useful for on-line range verification, studying the elemental composition of the tissue along the beam path, and studying blood flow within the tumor, all of which may have an impact on subsequent treatment planning and delivery.

In this work we present the measurements of the spatial and temporal distributions of positron-emitting activity created in tissue by continuous and pulsed proton beams at energies near $150 \mathrm{MeV}$. These measurements were made using a prototype imaging system designed for these preliminary investigations. The goals are: (i) to determine if such a system may be used as an accurate on-line range monitor between beam pulses; (ii) quantify the temporal and spatial activity distributions of the various PET isotopes; (iii) determine if tissue composition may be ascertained from the isotopic activity distributions for the purpose of improving subsequent treatment planning and delivery; and (iv) investigate the possibility of using such a system to monitor dose.

\section{MATERIALS AND METHODS}

In the following sections we describe the proton beam and tissue phantoms used during the experiments and give a brief description of the imaging system. We then present the algorithms used to separate, reconstruct, and quantify the depthdistributions for each of the major positron-emitting isotopes produced in experiments where decay data were collected. This is followed by a description of the continuous and macro-pulsed beam experiments conducted.

\section{A. Characteristics of the proton beam}

A beam of protons was produced at the National Superconducting Cyclotron Laboratory at Michigan State University (NSCL) using a K1200 isochronous cyclotron. The primary beam of ionized molecular hydrogen was accelerated with rf frequencies of $24.1036 \mathrm{MHz}$ which produced a micro-pulse structure in the beams. The beam then passed through a stripping foil to dissociate the molecular bond and produce free protons which were then focused to an average FWHM diameter of $1 \mathrm{~cm}$. After passing through a $0.0254 \mathrm{~cm}$ thick Havar vacuum window and into air, the beams entered the phantoms at $150 \mathrm{MeV}$.

\section{B. Tissue phantoms}

The $5 \times 5 \times 27 \mathrm{~cm}^{3}$ tissue phantoms were placed with their long dimension along the axis of the proton beam. The beam entered the phantom through the center of one of the 5
TABLE I. Elemental tissue compositions by percent mass.

\begin{tabular}{lccc}
\hline \multicolumn{1}{c}{ Element } & Swine & Muscle & Adipose \\
\hline Oxygen & 74.61 & 72.893 & 27.8 \\
Hydrogen & 9.80 & 10.2 & 11.4 \\
Carbon & 11.32 & 12.3 & 59.8 \\
Nitrogen & 2.52 & 3.5 & 0.7 \\
Sulfur & $<0.5$ & 0.5 & 0.1 \\
Chlorine & $\ldots$ & $\ldots$. & 0.1 \\
Potassium & 0.29 & 0.3 & \\
Phosphorus & 0.27 & 0.2 & 0.1 \\
Sodium & 0.21 & 0.08 & \\
Magnesium & 0.065 & 0.02 & \\
Calcium & 0.022 & 0.007 & \\
Iron & 0.0034 & $\ldots$ & \\
\hline \hline
\end{tabular}

$\times 5 \mathrm{~cm}^{2}$ faces of the sample. This provided ample pathlength to stop the $150 \mathrm{MeV}$ proton beam, whose range was $16.0 \mathrm{~cm}$, in the phantom. This also provided a significant amount of tissue beyond the Bragg peak to observe any production of PET isotopes by neutron activation beyond the Bragg peak.

Most tissue-equivalent materials have electron densities similar to those of human tissues. However, for these experiments it was necessary to use a phantom with the correct isotopic composition, thus providing activity distributions similar to those which would be produced in human tissue. The elemental composition of the swine-tissue phantoms used for these experiments are shown in Table I, along with the composition of human muscle and adipose tissue for comparison. The most abundant elements in tissue from which positron-emitting isotopes may be produced are oxygen, carbon, and nitrogen (see the Appendix). The phantoms were placed and irradiated along the axis of a prototype online monitoring system which was used to monitor the positron-emitting activity produced.

\section{The on-line imaging system}

The $\gamma$-ray detection system used consisted of two rows of BGO detectors as shown in Fig. 1. Each row contained 12 detectors. Each BGO detector measured $1.91 \mathrm{~cm}$ in diameter by $5.08 \mathrm{~cm}$ long and was separated from its neighbor by 0.51 $\mathrm{cm}$. The front surfaces of the detectors between the two rows were separated by $60 \mathrm{~cm}$.

Both rows of detectors were encased in custom-made lead shields to reduce background radiation and to suppress detection of Compton scattered $\gamma^{\prime}$ s and $\beta^{-}$'s between crystals. In addition, lead shutters provided shielding to the front of the detectors while the beam was on to avoid activation of the crystals. These shutters were operated by a pneumatic cylinder which was controlled by a variable time delay relay switch. A photogate mounted on the shutters controlled the phase of the rf to the cyclotron so the beam would be off when the shutters were open.

The detectors in one row were placed in coincidence with the detectors in the other row, resulting in 144 possible detector combinations for detecting positron decay events. However, many of the detector pairs monitor the same region along the beam path resulting in 23 unique monitored 


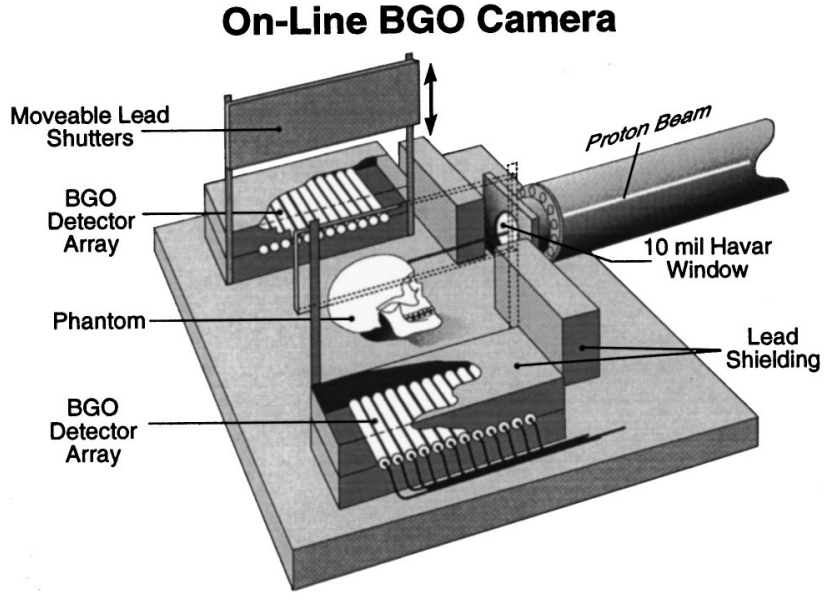

FIG. 1. Prototype on-line imaging system.

positions. These 23 resolvable positions are located between the 12 opposing detector pairs plus the 11 positions between neighboring detector pairs. The number of coincidence events detected in each of these 23 unique positions, due to a positron decay distribution, $\mathcal{D}$, constitutes the vector response, $\mathcal{R}$ of the system. The mapping between the two representations is provided by the system matrix, $\mathcal{S}$, which is measured during the calibration process.

The system matrix is formed by moving a calibrated positron-emitting source along the system axis and recording the response vector, $\mathcal{R}$, over each of the 23 unique positions. These response vectors are then placed beside each other in columns to form the system matrix. The matrix characterizes the solid angle, efficiency, detection probability, and accidental coincidence probability of the detection system at each of the 23 unique positions monitored by the 12 detector pairs. The total length of the system is $28.4 \mathrm{~cm}$, consequently, each of the 23 resolvable positions is approximately $1.2 \mathrm{~cm}$ in length. The imaging system has been described in greater detail in other works. ${ }^{33-35}$

\section{Image reconstruction and isotopic imaging}

The general image reconstruction algorithm for the system has been discussed in Ref. 33. Here we present the technique used to image individual radioisotopes produced in the field of view. The proton beam creates a distribution of PET activity, $\mathcal{A}(\mathbf{r}, t)$, as given by Eq. (A5). Over the image acquisition time, $T_{I}=t_{2}-t_{1}$, the spatial distribution of decays is

$$
\mathcal{D}(\mathbf{r})=\int_{t_{1}}^{t_{2}} \mathcal{A}(\mathbf{r}, t) d t .
$$

This vector distribution of decays produces a vector response, $\mathcal{R}$, from the imaging system. The vector response must be corrected for attenuation within the object to make quantitative measurements. In the results presented here, this was accomplished by calculating the pathlength through the phantom for each detector pair. Objects with more complicated geometries may require transmission studies with a calibration source to obtain attenuation corrections. The mapping between the vector response and the spatial decay distribution is given by the system matrix, $\mathcal{S} .{ }^{33}$ The spatial decay distribution is given by

$$
\mathcal{D}_{\mathrm{abs}}=\frac{1}{m}\left(\frac{A_{\mathrm{cal}} L}{\nu_{s}}\right) \mathcal{S}^{-1} \mathcal{R},
$$

where $A_{\text {cal }}$ is the activity of the calibration source in decays per second, $L$ is the length of the imaging array, $\nu_{S}$ is the velocity of the calibration source, $m$ is the number of elements in the reconstructed decay distribution, and $\mathcal{S}^{-1}$ is the inverse of the system matrix. During on-line imaging, data were acquired between beam pulses. In these experiments the image acquisition time was typically $5 \mathrm{~s}$ which is half the beam period, $T_{I}=T / 2$. After each beam pulse, the expected decay distribution based on Eqs. (A4) and (A5) may be compared with that found by Eq. (2).

Different isotopes may be imaged if a series of datasets are acquired during the decay of the activity distribution. This "radioisotopic imaging" is particularly useful for determining the distribution of oxygen along the beam path since the ${ }^{16} \mathrm{O}(p, n){ }^{15} \mathrm{O}$ reaction is the only significant source of ${ }^{15} \mathrm{O}$ in soft tissues and bone. The total imaging time should be about three times the half-life of the radioisotopes being examined, or 5 to $6 \mathrm{~min}$ in the case of ${ }^{15} \mathrm{O}$, to obtain the most events in the shortest amount of time and adequately sample the decay curve of interest.

Suppose the decaying activity distribution is continuously sampled by recording the response vectors in a series of time increments of length, $T_{I}=t_{2}-t_{1}$. In any given element of one of the response vectors, the number of counts is proportional to the sum of the isotopic activities at the observed position and has the form

$$
\begin{aligned}
& \mathcal{D}_{j} \propto \int_{t_{1}}^{t_{2}}\left[\sum_{i} \mathcal{A}_{i}^{\max } e^{-\lambda_{i} l}\right] d t, \\
& \mathcal{D}_{j} \propto \sum_{i} \mathcal{A}_{i}^{\max }\left(\frac{e^{-\lambda_{i} T_{l}-1}}{\lambda_{i}}\right) e^{-\lambda_{i} t_{2}} .
\end{aligned}
$$

The sequential decay data recorded for each element of the response vector may be fit to a decay model to find the initial distribution of activity, $\mathcal{A}_{i}^{\max }$, for each isotope in response space. Statistical tests for determining the presence of trace radioisotopes in the decay data are presented in the next section. Once the initial activity of each radioisotope has been determined, the total number of counts occurring in that element of the response vector due to a specific radioisotope may be found by integrating the decay curve with the appropriate constants. In this way isotopic response vectors may be obtained for each radioisotope occurring in the field of view of the imaging system. Radioisotopic images may then be found by using Eq. (2) with each of the radioisotopic response vectors.

\section{E. Error analysis and statistical tests}

The uncertainty in a reconstructed image may be found using the following theorem: ${ }^{36}$ 
"Let $\mathcal{D}$ be an $n$-dimensional normal random vector with positive definite covariance matrix $K$ and mean vector $\mu$. Let $\mathcal{S}$ be a nonsingular linear transformation in $n$ dimensions. Then $\mathcal{R}=\mathcal{S D}$ is an $n$-dimensional normal random vector with covariance matrix $Q$ $=\mathcal{S} K \mathcal{S}^{T} \ldots$,

The covariance matrix, $Q$, of the response vector, $\mathcal{R}$, contains the variance of the elements of $\mathcal{R}$ on its diagonal with all other elements of $Q$ being zero. The uncertainty in the elements of $\mathcal{R}$ is Poisson so that $Q_{j j}=\mathcal{R}_{j}$. The counting of radioactive isotopes is also a Poisson process, therefore the variance in the elements of the reconstructed image is given by the diagonal elements of the covariance matrix, $K$, where $K$ is given by

$$
K=\mathcal{S}^{-1} Q\left(\mathcal{S}^{T}\right)^{-1} \text {. }
$$

The uncertainties in radioisotopic images are compounded by the uncertainty in determining the initial activity coefficients while fitting the decay data. The decay curves are fit using the Levenberg-Marquardt method with Poisson weighting. While this nonlinear fitting algorithm may be used to determine the half-lives of unknown radioisotopes, the half-lives of the isotopes produced in tissue are well known. In this study only the linear activity coefficients are included in the decay model while the half-lives are fixed. This algorithm calculates the covariance matrix for the fit parameters, giving an estimate of the uncertainty in each of the initial isotopic activities for each element of the response vector. The calculated variance is larger than the variance expected from Poisson counting statistics alone and also includes the variance in the fit. It is thus possible to form response variance vectors, $\mathcal{V}$, for each decaying radioisotope by integrating the decay curve with the appropriate constants. As described above, the covariance matrix for a given isotopic response vector is then $Q_{j j}=\mathcal{V}_{j}$.

The decay data from an experiment using a tissue phantom will predominately contain ${ }^{11} \mathrm{C},{ }^{13} \mathrm{~N}$, and ${ }^{15} \mathrm{O}$, but other positron-emitting isotopes such as ${ }^{10} \mathrm{C}$ and ${ }^{14} \mathrm{O}$ may be produced in smaller quantities. If the decay data from each of the 23 resolvable elements are summed, the statistics may be sufficient to estimate the abundances of these radioisotopes by including them in the fit to the summed decay data. Two statistical tests, based on the $F$-distribution, are used to validate the inclusion of trace radioisotopes in the decay model during a fit. ${ }^{37}$ The first is an $F$ test of the multiple-correlation coefficient, $\mathcal{R}$, and verifies that the fit coefficients are positive and tests the minimum square deviation of the data from the decay model. The second $F$ test compares two decay models which differ by one term, that of the trace radioisotope in question. The latter test is based on the difference in the chi-squares of the two decay models. The presence of the trace radioisotope in question may then be verified to the desired level of confidence, which in this work is set at $99 \%$. More details on these tests may be found in Ref. 37.

When the decay data from each of the resolvable elements are not summed, the statistics may not be sufficient to identify the trace radioisotopes present in any one of the ob- served segments along the system axis. The above tests are applied to obtain the optimal fit for each position. If the presence of the trace radioisotopes cannot be confirmed by the $F$ tests at the $99 \%$ confidence level, the trace radioisotope activity coefficients are set to zero. Measurements of the background rates in the imaging system resulted in vanishingly small rates. $F$ tests on the inclusion of a background term in the decay model indicate that it generally does not improve the fit of the data to the model. Including terms for background or trace radioisotopes in the decay model at significantly lower confidence levels introduces additional linear correlations among the isotopic activity coefficients which degrades the quality of the isotopic images.

\section{F. Continuous proton beam into a tissue phantom}

Using the materials and techniques described above, the production and distribution of short-lived isotopes was studied using a continuous beam. The activity was created by a $150 \mathrm{MeV}$ proton beam with a current of $1.75 \mathrm{nA}$ and an expected range of $16.0 \mathrm{~cm}$ in tissue. This beam was implanted continuously for $1 \mathrm{~min}$ into a swine-tissue phantom. The spatial and temporal decay distributions were measured beginning immediately after the beam was turned off. The surface of the $5 \times 5 \times 27 \mathrm{~cm}^{3}$ phantom was placed 2.0 $\pm 0.3 \mathrm{~cm}$ from the front end of the detector array, inside the imaging system on the systems axis. The beam was terminated by opening the shutters, which dephased the accelerating rf and enabled data acquisition. Decay data were collected for $57 \mathrm{~min}$ in $10 \mathrm{~s}$ bins. The initial coincidence rate was $2322 \mathrm{~Hz}$ with the computer being live for 2308 of these events giving an acquisition livetime of $99.4 \%$. The total number of coincidence events recorded was $9.07 \times 10^{5}$ with $7.92 \times 10^{5}$ of these meeting the required timing and energy window cuts. The initial singles rates in each of the 24 detectors, after the beam was turned off, ranged from $3500 \mathrm{~Hz}$ to $6700 \mathrm{~Hz}$.

\section{G. Macro-pulsed proton beams into tissue phantoms}

Variable macro-pulse periods were achieved by phasing and dephasing the rf to the cyclotron dees in synchronization with the pneumatically controlled shutter system on the imaging system. The period of the shutters was controlled by a variable time delay relay switch. A photogate mounted on the shutters controlled the phase of the rf to the cyclotron so the beam would be off when the shutters were open. Typical periods for a shutter cycle were $10 \mathrm{~s}$.

The feasibility of imaging between beam macro-pulses was investigated by performing experiments using a 150 $\mathrm{MeV}$ proton beam with a current of $1.75 \mathrm{nA}$, macro-pulsed with a $10 \mathrm{~s}$ period. This beam was implanted into a tissue phantom for 2 min resulting in $12,5 \mathrm{~s}$ beam pulses being delivered to the phantom. Data were acquired for $5 \mathrm{~s}$ between each of the 12 beam pulses and in $10 \mathrm{~s}$ bins for $56 \mathrm{~min}$ after beam delivery. A swine-tissue phantom was placed with its front surface $6.0 \pm 0.3 \mathrm{~cm}$ inside the imaging system.

Another pulsed-beam experiment was conducted to investigate the maximum activity produced with pulsed beams for 

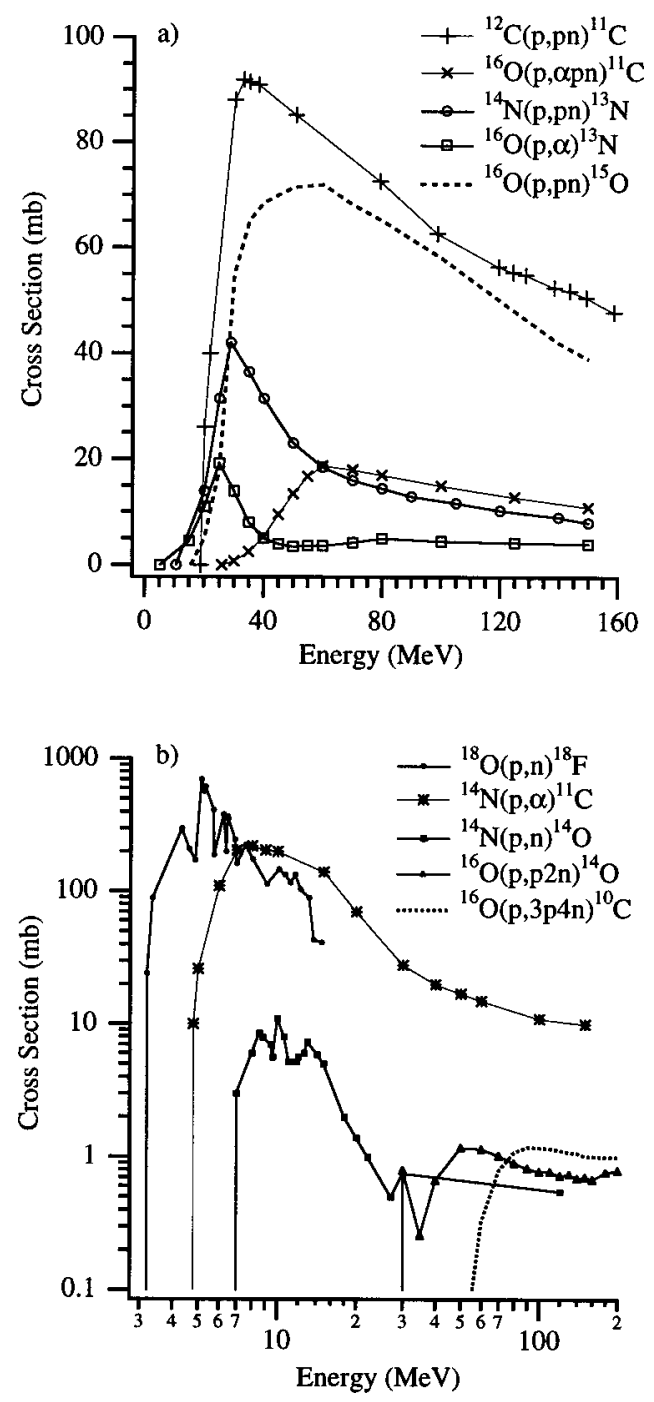

FIG. 2. (a) Cross sections for nuclear reactions leading to the production of positron-emitting materials. (b) Cross sections for nuclear reactions leading to the production of positron-emitting materials.

radioisotopes with half-lives comparable to or shorter than the treatment time. In this experiment a $0.14 \mathrm{nA}$ beam with a $20 \mathrm{~s}$ period was implanted into a tissue phantom for $21 \mathrm{~min}$. Data were acquired for $10 \mathrm{~s}$ between beam pulses and continued for an additional $61 \mathrm{~min}$ after the beam ended. The front surface of the $5 \times 5 \times 27 \mathrm{~cm}^{3}$ swine-tissue phantom was placed $2.0 \pm 0.3 \mathrm{~cm}$ inside the front end of the imaging system.

\section{RESULTS}

The following sections contain the results of several imaging experiments carried out using continuous and pulsed protons beams implanted into tissue phantoms. Decay data were collected for the three experiments presented. In each case, images are presented based on the cumulative dataset and based on the isotopic imaging technique described earlier. These are compared with the expected decay distributions as calculated using the reaction cross sections shown in Figs. 2(a) and 2(b) and the composition shown in Table I

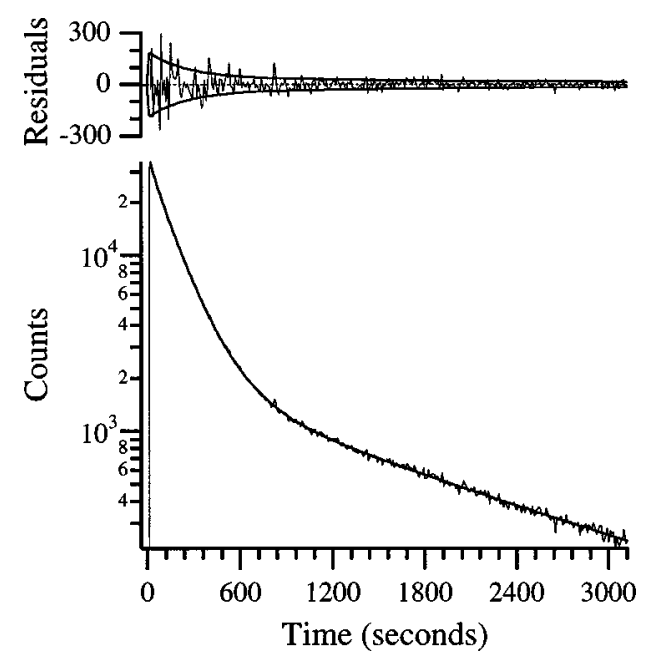

FIG. 3. The total decay data collected while imaging after a $1.75 \mathrm{nA}, 150$ $\mathrm{MeV}$ proton beam was implanted for $60 \mathrm{~s}$. The fit to the data shows that ${ }^{15} \mathrm{O}$, ${ }^{14} \mathrm{O},{ }^{13} \mathrm{~N},{ }^{11} \mathrm{C}$, and ${ }^{10} \mathrm{C}$ are present at the $99 \%$ confidence level. The residuals of this fit are shown at the top with the Poisson uncertainty.

which is assumed to be uniform. In the case of macro-pulsed beams, images acquired between beam pulses are presented.

\section{A. Continuous proton beams into a tissue phantom}

Figure 3 shows the sum of the decay data from the 23 positions, the fit to the decay data, and the residuals which are shown at the top with the Poisson uncertainty. The best fit to the decay data is obtained by including ${ }^{15} \mathrm{O},{ }^{14} \mathrm{O},{ }^{13} \mathrm{~N}$, ${ }^{11} \mathrm{C}$, and ${ }^{10} \mathrm{C}$. The $F$ tests for a decay model including ${ }^{14} \mathrm{O}$ and ${ }^{10} \mathrm{C}$ indicate both are present at a $99 \%$ confidence level in the summed decay data. However, including a background term is not justified by these tests and this coefficient was set to zero. The isotopic response vectors shown in Fig. 4 were produced by first fitting the decay data to the above decay model for the individual "pixels." The validity of including the ${ }^{10} \mathrm{C}$ term in the decay model was tested for each set of decay data but was not justified at the $99 \%$ confidence level for any of the pixels. Figure 4 shows the cumulative and radioisotopic response vectors integrated over the imaging

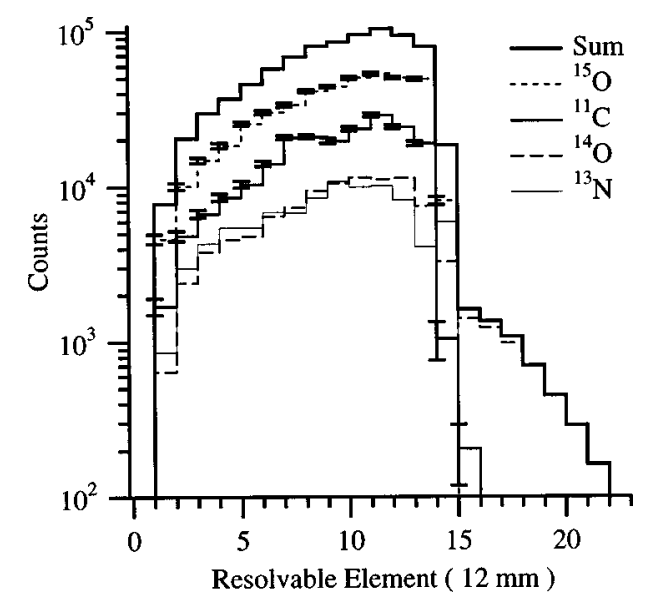

FIG. 4. Radioisotopic response vectors generated from decay data. 


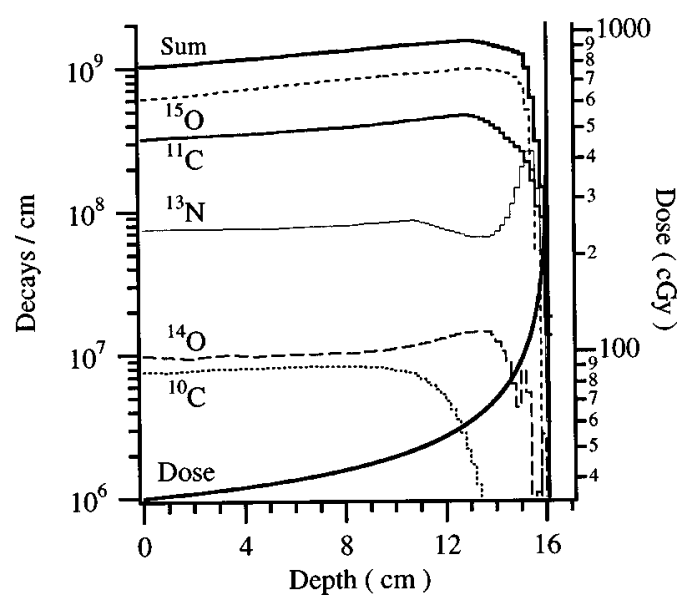

FIG. 5. Expected isotopic decay distribution for a $1.75 \mathrm{nA}, 150 \mathrm{MeV}$ proton beam implanted for $60 \mathrm{~s}$.

time but before image reconstruction. The error bars shown are the square root of the elements of the variance vectors, $\sqrt{\mathcal{V}_{j}}$, described above. The error bars were omitted for the ${ }^{14} \mathrm{O}$ and ${ }^{13} \mathrm{~N}$ response vectors for illustrative purposes but are comparable to the others shown. Figure 5 shows the expected activity distribution where different reactions leading to the same radioisotope have been added. The radioisotopic image vectors, shown in Fig. 6, were calculated using Eq. (2). The range of the $150 \mathrm{MeV}$ proton beam is $16.0 \mathrm{~cm}$ in tissue and is well determined within the resolution of the imaging system. The distribution of activity drops abruptly near the end of the proton beams range as the proton energy falls below the threshold energies of the various nuclear reactions. However, some slight but significant ${ }^{14} \mathrm{O}$ activity is observed beyond this range.

Table II gives the activity expected for each reaction, the expected total activity; and number of decays, while imaging, by radioisotope, and the total number of decays as de-

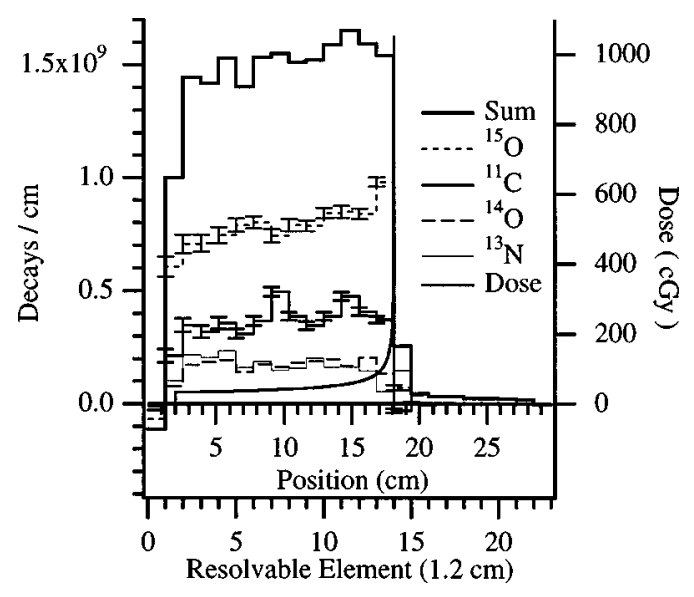

FIG. 6. Radioisotopic images of the decay distributions of activity created by a $1.75 \mathrm{nA}, 150 \mathrm{MeV}$ proton beam implanted for $60 \mathrm{~s}$.

termined from the reconstructed isotopic images. The uncertainties given with the experimentally determined values only reflect the uncertainties due to counting statistics and fitting the data to the decay model. The uncertainty in the beam current during the experiments was about 20\%. (During an actual treatment the beam current would be monitored to a much higher precision using a transmission ionization chamber.) Given these uncertainties, the abundance and distribution of ${ }^{11} \mathrm{C}$ of ${ }^{15} \mathrm{O}$ agree well with the expected distributions. However, based on the known tissue composition, the amount of ${ }^{13} \mathrm{~N}$ observed is almost twice as large as expected and the observed amount of ${ }^{14} \mathrm{O}$ is over 17 times the expected amount.

\section{B. Pulsed proton beams into a tissue phantom}

The production and decay of the activity resulting from a 2 min macro-pulsed beam with a 10 s period is shown in Fig. 7. As expected, the number of coincidences detected between

TABLE II. Summary of the isotopic activities produced and the expected and measured number of total coincidence events. This activity was produced by a $1.75 \mathrm{nA}, 150 \mathrm{MeV}$ proton beam which was implanted into a swine-tissue phantom continuously for $60 \mathrm{~s}$.

\begin{tabular}{lcccc}
\hline \hline \multicolumn{1}{c}{ Reaction } & $\begin{array}{c}\text { Expected } \\
\text { activity }(\mu \mathrm{Ci}) \\
\text { by reaction }\end{array}$ & $\begin{array}{c}\text { Expected } \\
\text { activity }(\mu \mathrm{Ci}) \\
\text { by isotope }\end{array}$ & $\begin{array}{c}\text { Expected } \\
\text { counts }\left(\times 10^{9}\right) \\
\text { by isotope }\end{array}$ & $\begin{array}{c}\text { Measured } \\
\text { counts }\left(\times 10^{9}\right) \\
\text { by isotope }\end{array}$ \\
\hline${ }^{16} \mathrm{O}(p, 3 p 4 n){ }^{10} \mathrm{C}$ & 94.0 & 94.0 & 0.097 & \\
${ }^{12} \mathrm{C}(p, p n){ }^{11} \mathrm{C}$ & 52.0 & 107.8 & 5.8 & $5.74 \pm 0.08$ \\
${ }^{14} \mathrm{~N}(p, \alpha){ }^{11} \mathrm{C}$ & 2.6 & & & \\
${ }^{16} \mathrm{O}(p, \alpha p n){ }^{11} \mathrm{C}$ & 53.2 & 43.3 & 1.3 & $2.74 \pm 0.09$ \\
${ }^{14} \mathrm{~N}(p, p n){ }^{13} \mathrm{~N}$ & 4.4 & 42.9 & 0.162 & $2.91 \pm 0.09$ \\
${ }^{16} \mathrm{O}(p, \alpha){ }^{13} \mathrm{~N}$ & 38.9 & & & $12.31 \pm 0.11$ \\
${ }^{14} \mathrm{~N}(p, n){ }^{14} \mathrm{O}$ & 1.1 & 1877.5 & & \\
${ }^{16} \mathrm{O}(p, p 2 n){ }^{14} \mathrm{O}$ & 41.8 & 0.0023 & 19.61 & $23.70 \pm 0.19$ \\
${ }^{16} \mathrm{O}(p, p n){ }^{15} \mathrm{O}$ & 1877.5 & 2248.8 & & \\
${ }^{18} \mathrm{O}(p, n){ }^{18} \mathrm{~F}$ & 0.0023 & & & \\
$\mathrm{Totals}$ & &
\end{tabular}




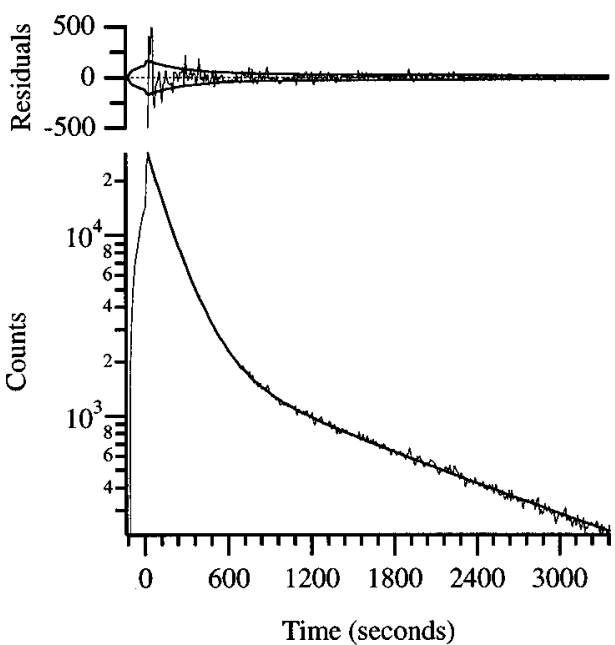

FIG. 7. The total decay data collected while imaging after a $1.75 \mathrm{nA}, 150$ $\mathrm{MeV}$ proton beam with a $10 \mathrm{~s}$ pulse period was implanted for $120 \mathrm{~s}$. Statistical tests of the decay model show that ${ }^{15} \mathrm{O},{ }^{14} \mathrm{O},{ }^{13} \mathrm{~N},{ }^{11} \mathrm{C}$, and ${ }^{10} \mathrm{C}$ are present at the $99 \%$ confidence level. The residuals of this fit are shown at the top with the Poisson uncertainty.

beam pulses increases approximately linearly with total irradiation time on short time scales. During a patient treatment, verifying the range of the beam early in the treatment is of primary importance. Ideally, using a macro-pulsed beam, the range could be measured after one pulse with the continuation of the treatment being contingent upon verification of the proper range. Figure 8 shows the reconstructed image after one macro-beam pulse. This image was reconstructed from 1431 coincidence events. The expected dose distribution is shown for comparison. The uncertainties in the image produced after one pulse are large, nonetheless the number of events is adequate for range verification.

Inspection of the inside of the swine-tissue phantom revealed a small adipose deposit beginning $11.2 \pm 0.1 \mathrm{~cm}$ from the surface of the phantom. Its spatial extent along the beam path was $0.4 \pm 0.1 \mathrm{~cm}$. The elemental composition of this material was assumed to be similar to that given in Ref. 38

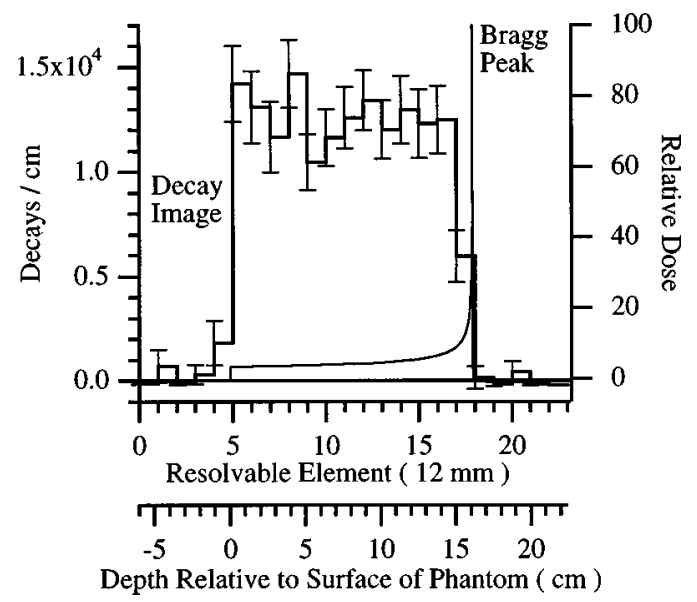

FIG. 8. Range verification using the reconstructed decay distribution after one macro-beam pulse. This distribution was reconstructed from $1431 \mathrm{co}-$ incidence events. The expected dose distribution is shown for comparison.

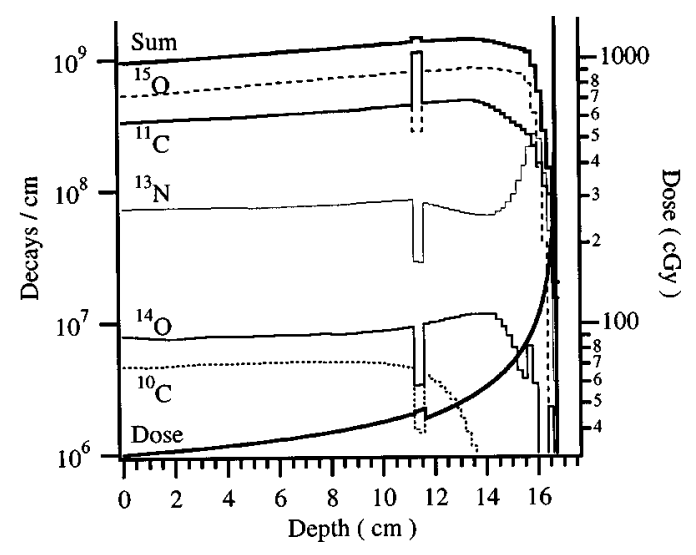

FIG. 9. Expected isotopic decay distribution over $56 \mathrm{~min}$ for the activity created by a $1.75 \mathrm{nA}, 150 \mathrm{MeV}$ proton beam with a $10 \mathrm{~s}$ period which was implanted in a tissue phantom for $120 \mathrm{~s}$. The dose distribution for a monoenergetic beam is shown for range comparison.

and is shown in Table I. The decay distribution of positronemitting activity, given this phantom geometry and composition, is shown in Fig. 9. The presence of the adipose tissue changes the range of the beam by a small amount which is well below the resolution of the imaging system. Consequently, we do not experimentally observe a deviation in the proton range as might be expected. The decay distribution in Fig. 9 shows a slightly larger number of decays from the adipose region. This is due to the long half-life of ${ }^{11} \mathrm{C}$ and the long, 56 min, imaging time. The initial amount of ${ }^{15} \mathrm{O}$ activity in this adipose region is about half that of regions containing muscle tissue due to the differing oxygen content.

As in the experiments with continuous beams, ${ }^{15} \mathrm{O},{ }^{14} \mathrm{O}$, ${ }^{13} \mathrm{~N},{ }^{11} \mathrm{C}$, and ${ }^{10} \mathrm{C}$ are present in the summed decay data at the $99 \%$ confidence level. Radioisotopic response vectors were produced by first fitting the decay data to a decay model containing these radioisotopes for the individual pixels. Again, the validity of including the ${ }^{10} \mathrm{C}$ term in the decay model was tested for each set of decay data but was not justified at the $99 \%$ confidence level for any of the pixels. The isotopic images are shown in Fig. 10. Note the dip in the

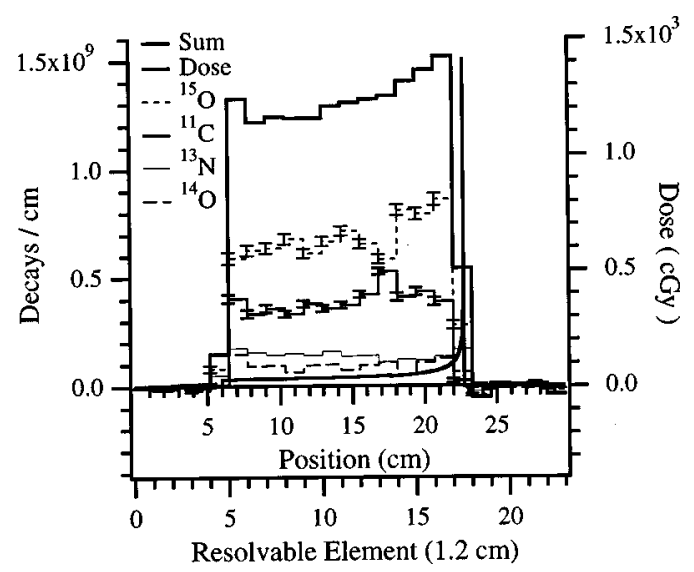

FIG. 10. Radioisotopic images of the decay distributions of activity created by a $1.75 \mathrm{nA}, 150 \mathrm{MeV}$ proton beam with a $10 \mathrm{~s}$ period which was implanted in a tissue phantom for $120 \mathrm{~s}$. 
TABLE III. Summary of the isotopic activities produced and the expected and measured number of total coincidence events. This activity was produced by a $1.75 \mathrm{nA}, 150 \mathrm{MeV}$ proton beam which was pulsed with a $10 \mathrm{~s}$ period and implanted into a swine-tissue phantom for $120 \mathrm{~s}$.

\begin{tabular}{lcccc}
\hline \hline Reaction & $\begin{array}{c}\text { Expected } \\
\text { activity }(\mu \mathrm{Ci}) \\
\text { by reaction }\end{array}$ & $\begin{array}{c}\text { Expected } \\
\text { activity }(\mu \mathrm{Ci}) \\
\text { by isotope }\end{array}$ & $\begin{array}{c}\text { Expected } \\
\text { counts }\left(\times 10^{9}\right) \\
\text { by isotope }\end{array}$ & $\begin{array}{c}\text { Measured } \\
\text { counts }\left(\times 10^{9}\right) \\
\text { by isotope }\end{array}$ \\
\hline${ }^{16} \mathrm{O}(p, 3 p 4 n){ }^{10} \mathrm{C}$ & 56.2 & 56.2 & 0.058 & \\
${ }^{12} \mathrm{C}(p, p n){ }^{11} \mathrm{C}$ & 57.1 & 110.9 & 6.15 & $5.70 \pm 0.06$ \\
${ }^{14} \mathrm{~N}(p, \alpha){ }^{11} \mathrm{C}$ & 2.5 & & & \\
${ }^{16} \mathrm{O}(p, \alpha p n){ }^{11} \mathrm{C}$ & 51.3 & 41.3 & 1.29 & $2.28 \pm 0.07$ \\
${ }^{14} \mathrm{~N}(p, p n){ }^{13} \mathrm{~N}$ & 4.2 & 33.6 & 0.13 & $1.61 \pm 0.07$ \\
${ }^{16} \mathrm{O}(p, \alpha){ }^{13} \mathrm{~N}$ & 37.1 & & 10.42 & $10.19 \pm 0.09$ \\
${ }^{14} \mathrm{~N}(p, n){ }^{14} \mathrm{O}$ & 0.85 & 1597.6 & & $19.78 \pm 0.15$ \\
${ }^{16} \mathrm{O}(p, p 2 n){ }^{14} \mathrm{O}$ & 32.7 & 0.00236 & 18.05 & \\
${ }^{16} \mathrm{O}(p, p n){ }^{15} \mathrm{O}$ & 1597.6 & 1910.1 & & \\
${ }^{18} \mathrm{O}(p, n){ }^{18} \mathrm{~F}$ & 0.00236 & & & \\
$\mathrm{Totals}$ & & &
\end{tabular}

${ }^{15} \mathrm{O}$ distribution and the rise in the ${ }^{11} \mathrm{C}$ distribution due to the adipose deposit in the thirteenth resolvable element. Table III gives the total activity expected for each reaction, the expected total activity by radioisotope, and the expected and measured number of decays by radioisotope. The uncertainty in the expected activities given in Table III due to the uncertainty in the beam current is $20 \%$. The amount of ${ }^{15} \mathrm{O}$ and ${ }^{11} \mathrm{C}$ agree well within these uncertainties. However, the amount of ${ }^{13} \mathrm{~N}$ is $77 \%$ larger than expected and the amount of ${ }^{14} \mathrm{O}$ is 12.4 times larger than expected.

\section{Activity saturation with macro-pulsed beams in tissue}

The production of PET activity due to a large number of pulses, and subsequent decay, is shown in Fig. 11, where time zero corresponds to the end of the irradiation. The production of activity is relatively constant over the first 2-3 min of irradiation while the abundance of ${ }^{15} \mathrm{O}$ is still low. The sudden decrease in the number of counts between pulses which occurs at $-500 \mathrm{~s}$ is due to small variations in the response time of the shutter system relative to that of the data acquisition system. It can be seen that after $20 \mathrm{~min}$ the production rate has slowed considerably as all isotopes decay almost as quickly as they are produced. Since the beam duration is comparable to the half-life of ${ }^{11} \mathrm{C}, 20 \mathrm{~min}$, and ${ }^{13} \mathrm{~N}$, $10 \mathrm{~min}$, the activity of these isotopes will not be at their maximum levels. The activities of isotopes with half-lives shorter than roughly $5 \mathrm{~min}$, such as ${ }^{15} \mathrm{O}$ and ${ }^{14} \mathrm{O}$, should be near their maximum levels. The decay data shown in Fig. 11 was fit and found to contain primarily ${ }^{15} \mathrm{O},{ }^{13} \mathrm{~N},{ }^{11} \mathrm{C}$, and some ${ }^{14} \mathrm{O} .{ }^{10} \mathrm{C}$ is not present at the $95 \%$ confidence level in the summed decay data. Again, while ${ }^{14} \mathrm{O}$ is present at the 99\% confidence level in the total decay data, it was not found at this level in any of the individual positions. Fitting for ${ }^{15} \mathrm{O},{ }^{13} \mathrm{~N}$, and ${ }^{11} \mathrm{C}$ and reconstructing their decay distributions gives the radioisotopic images shown in Fig. 12. The expected activity distributions, where different reactions leading to the same radioisotope have been added, are shown in Fig. 13. Note that under the given beam and imaging parameters, the number of decays from ${ }^{11} \mathrm{C}$ is greater than from ${ }^{15} \mathrm{O}$. This is due to the $21 \mathrm{~min}$ beam duration. Table IV gives the total activity expected for each reaction, the expected total activity by radioisotope, and the expected and measured number of decays while imaging by radioisotope. Again, the uncertainty in the total expected activity and expected counts by isotope is about $20 \%$ due to the uncertainty in the beam current. As before, the measured amounts of ${ }^{11} \mathrm{C}$ and ${ }^{15} \mathrm{O}$ agree with the expected amounts but the measured amount of ${ }^{13} \mathrm{~N}$ is twice as high as expected.

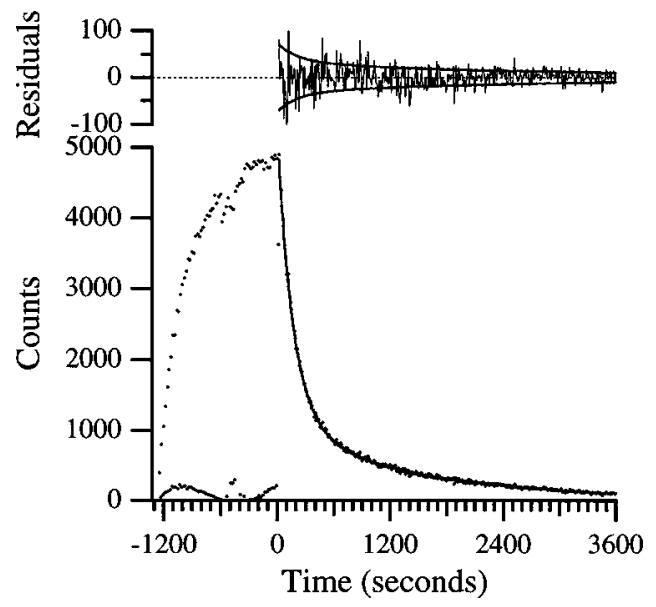

FIG. 11. The total decay data collected while imaging after a $0.14 \mathrm{nA}, 150$ $\mathrm{MeV}$ proton beam with a $20 \mathrm{~s}$ pulse period was implanted for $23 \mathrm{~min}$. Statistical tests of the decay model shows that ${ }^{15} \mathrm{O},{ }^{14} \mathrm{O},{ }^{13} \mathrm{~N}$, and ${ }^{11} \mathrm{C}$ are present at the $99 \%$ confidence level. The fit to the decay data using this model is shown by the solid line and the residuals of this fit are shown at the top with the Poisson uncertainty. 


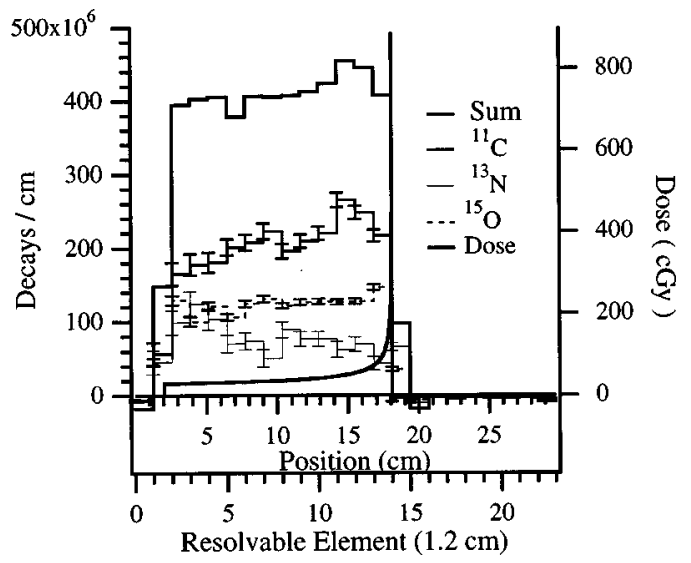

FIG. 12. Radioisotopic images of the decay distributions of ${ }^{15} \mathrm{O},{ }^{13} \mathrm{~N}$, and ${ }^{11} \mathrm{C}$ activity created by a $0.14 \mathrm{nA}, 150 \mathrm{MeV}$ proton beam with a $20 \mathrm{~s}$ period which was implanted in a tissue phantom for $23 \mathrm{~min}$.

\section{DISCUSSION}

\section{A. Imaging system design}

It has been shown that the range of a proton therapy beam can be verified on-line within the resolution of the imaging system and some information on composition can be obtained. However, several issues still need to be addressed. To be optimal for clinical use the resolution of the imaging system must be reduced to the subcentimeter range. In designing a detection system to accomplish this, consideration must be given to the average coincidence detection efficiency per unit length along the system axis. To maintain sufficient efficiency to obtain statistically significant images, the detector height perpendicular to the plane of the detector array would have to increase if the width of each detector is decreased. This increase in detector volume would also increase the chance coincidence rate, especially if multiple crystals are optically coupled and fewer PMT's used for readout. How-

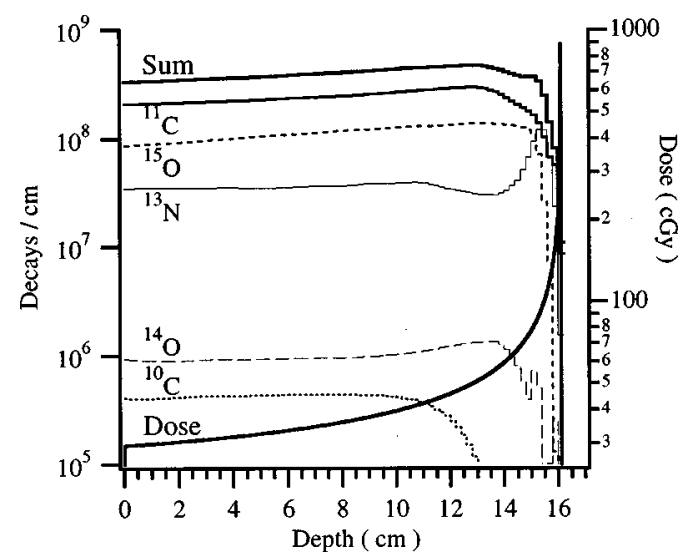

FIG. 13. Expected isotopic decay distribution over $61 \mathrm{~min}$ for the activity created by a $0.14 \mathrm{nA}, 150 \mathrm{MeV}$ proton beam with a $20 \mathrm{~s}$ period which was implanted in a tissue phantom for $23 \mathrm{~min}$. The dose distribution for a monoenergetic beam is shown for range comparison.

ever, the detector volume could be reduced by shortening the length of the BGO crystals from $5 \mathrm{~cm}$ to $3 \mathrm{~cm}$ with little loss of detection efficiency. Further design considerations and suggested improvements for on-line PET isotope monitoring devices may be found in Ref. 33 .

\section{B. Nuclear secondaries and the production of ${ }^{14} \mathrm{O}$}

The discrepancies noted above, between the amount of ${ }^{14} \mathrm{O}$ expected and the amount observed, may arise from at least two different sources. The calculated and experimental cross sections used to calculate the production of ${ }^{14} \mathrm{O}$ have some uncertainty ${ }^{39,40}$ and ${ }^{14} \mathrm{O}$ may be produced in nuclear reactions induced by secondary particles such as neutrons. The cross sections used to calculate the amount of ${ }^{14} \mathrm{O}$ created by the ${ }^{16} \mathrm{O}(p, p 2 n){ }^{14} \mathrm{O}$ reaction were calculated using the GNASH code system. ${ }^{41}$ These calculated cross sections are about a factor of 2 smaller than experimental values which

TABLE IV. Summary of the isotopic activities produced and the expected and measured number of total coincidence events. This activity was produced by a $0.14 \mathrm{nA}, 150 \mathrm{MeV}$ proton beam which was pulsed with a $20 \mathrm{~s}$ period and implanted into a swine-tissue phantom for $21 \mathrm{~min}$.

\begin{tabular}{lcccc}
\hline \hline Reaction & $\begin{array}{c}\text { Expected } \\
\text { activity }(\mu \mathrm{Ci}) \\
\text { by reaction }\end{array}$ & $\begin{array}{c}\text { Expected } \\
\text { activity }(\mu \mathrm{Ci}) \\
\text { by isotope }\end{array}$ & $\begin{array}{c}\text { Expected } \\
\text { counts }\left(\times 10^{9}\right) \\
\text { by isotope }\end{array}$ & $\begin{array}{c}\text { Measured } \\
\text { counts }\left(\times 10^{9}\right) \\
\text { by isotope }\end{array}$ \\
\hline${ }^{16} \mathrm{O}(p, 3 p 4 n){ }^{10} \mathrm{C}$ & 5.01 & 5.01 & 0.0052 & \\
${ }^{12} \mathrm{C}(p, p n){ }^{11} \mathrm{C}$ & 31.83 & & 3.75 & $3.08 \pm 0.04$ \\
${ }^{14} \mathrm{~N}(p, \alpha){ }^{11} \mathrm{C}$ & 1.57 & 65.97 & & $1.25 \pm 0.05$ \\
${ }^{16} \mathrm{O}(p, \alpha p n){ }^{11} \mathrm{C}$ & 32.57 & 19.94 & 0.63 & \\
${ }^{14} \mathrm{~N}(p, p n){ }^{13} \mathrm{~N}$ & 2.04 & & & \\
${ }^{16} \mathrm{O}(p, \alpha){ }^{13} \mathrm{~N}$ & 17.90 & 4.05 & 0.015 & $1.88 \pm 0.02$ \\
${ }^{14} \mathrm{~N}(p, n){ }^{14} \mathrm{O}$ & 0.10 & & & \\
${ }^{16} \mathrm{O}(p, p n){ }^{14} \mathrm{O}$ & 3.95 & 267.65 & & 6.75 \\
${ }^{16} \mathrm{O}(p, p n){ }^{15} \mathrm{O}$ & 267.65 & 0.0019 & 6.15 & \\
${ }^{18} \mathrm{O}(p, n){ }^{18} \mathrm{~F}$ & 0.0019 & 362.62 & & \\
$\mathrm{Totals}$ & & &
\end{tabular}


have been reported. ${ }^{42}$ In addition, a significant number of neutrons and other light, energetic charged secondary particles, including protons and $\alpha$-particles, are created which may induce secondary nuclear reactions. ${ }^{20}$ The production of positron-emitting isotopes apparently by secondary neutrons is evident beyond the protons range in Fig. 4. This activity is almost entirely ${ }^{14} \mathrm{O}$ and is most likely produced by the ${ }^{16} \mathrm{O}(n, 3 n){ }^{14} \mathrm{O}$ reaction which has threshold energy of 30.7 $\mathrm{MeV}$. (Further details on the dose and activity created by nuclear secondaries may be found in Refs. 20, 35.)

The effects of nuclear interactions on the dose distribution have been presented by other investigators. ${ }^{20,21}$ However, the effect of nuclear secondaries on the activity distribution has not been reported in detail yet. The radioisotopic images suggest that a library of accurate cross sections over a range of energies needs to be developed for neutron- and protoninduced reactions on the elements in tissue. ${ }^{40,43,44}$ The presence of activity beyond the proton-beam range suggests that nuclear reactions induced by secondary neutrons and other energetic secondary charged particles may produce a significant amount of ${ }^{14} \mathrm{O}$ in the treatment volume. However, since ${ }^{10} \mathrm{C},{ }^{13} \mathrm{~N}$, and ${ }^{14} \mathrm{O}$ are only produced in small quantities, the uncertainties in their cross sections are of little consequence to on-line range verification. Most tissues are primarily composed of oxygen, carbon, and hydrogen for which the cross sections for producing positron-emitting radioisotopes are reasonably well known. Although the unexpectedly large production of ${ }^{14} \mathrm{O}$ may have a noticeable effect on the magnitude of images produced on short time scales, due to its short half-life, it is probably produced from oxygen through the ${ }^{16} \mathrm{O}(p, p 2 n){ }^{14} \mathrm{O}$ and ${ }^{16} \mathrm{O}(n, 3 n){ }^{14} \mathrm{O}$ reactions. Thus the ${ }^{14} \mathrm{O}$ distribution primarily reflects the distribution of oxygen along the beam path.

Unlike ${ }^{14} \mathrm{O}$, there are no neutron-induced reactions leading to the production of ${ }^{13} \mathrm{~N}$ whose cross sections are large enough to account for the discrepancies observed. This is supported by the lack of ${ }^{13} \mathrm{~N}$ beyond the range of the proton beam. Factors which may contribute to the discrepancy include uncertainties in the cross sections, beam current, and composition of the tissue.

\section{The effects of in vivo biological washout and the presence of ${ }^{14} \mathrm{O}$ on total dose monitoring}

As shown in earlier sections, the number of events detected between pulses $n$ and $(n+1)$ varies nearly linearly on time scales less than about $2 \mathrm{~min}$. While this is true in the experiments presented here, additional complications will arise in living tissue. Much of the ${ }^{15} \mathrm{O}$ created will be bound in water which freely diffuses through cell membranes. This activity is consequently carried out of the field of view by the tumor's vasculature. In this situation, the decay of ${ }^{15} \mathrm{O}$ may be roughly modeled by two exponential terms. ${ }^{10}$ The first term models the nonmobile fraction of the ${ }^{15} \mathrm{O}$ activity which disappears solely through radioactive decay. The second term models the mobile fraction of the ${ }^{15} \mathrm{O}$ activity which disappears through radioactive decay and in vivo biological washout. The decay constant of the second term depends on the half-life of ${ }^{15} \mathrm{O}$ and the average rate of blood flow through the imaged volume. ${ }^{10}$ The corresponding half-life for the washout term may vary greatly depending on the average blood flow rate. Reported values of the washout half-life range from roughly $40 \mathrm{~s}^{9}$ to over $3 \mathrm{~min} .{ }^{10}$ If the treatment volume being imaged has a high blood flow then the activity will appear to decay on shorter time scales. The linear approximation of the number of counts detected between beam pulses will become less accurate making it a less reliable indicator of dose received in well-perfused treatment volumes.

It should be noted that a standard water tank, typically used to verify the range and the dose distribution in many treatment facilities, is not suitable for calibrating this type of imaging system. The mechanisms which lead to the transport of activity within the tank are difficult to quantify accurately and may vary significantly from calibration to calibration. Typically, the amount of ${ }^{15} \mathrm{O}$ present is severely underdetermined due to these processes and the amount of ${ }^{14} \mathrm{O}$ is overestimated due to the difficulty in quantifying the transport mechanisms for the mobile component of the ${ }^{15} \mathrm{O}$. In the presence of these transport phenomena the ${ }^{15} \mathrm{O}$ radioisotopic images were observed to be rather strongly peaked at the distal end of the protons range, much like the Bragg peak.

\section{Correlation between the dose distributions and the induced activity distribution}

The change in the total dose deposited in the treatment volume as a function of time may be monitored fairly reliably in treatment volumes with low blood flow rates. Figure 11 shows that the number of $511 \mathrm{keV}$ gamma-ray pairs detected between beam pulses increases nearly linearly with the number of beam pulses, and consequently with the dose, for times less than about $2 \mathrm{~min}$. However, as mentioned earlier, it is difficult to infer the dose distribution from the activity distribution since the physical processes leading to the loss of energy and the creation of positron-emitting nuclei are not directly related. In the experimental results sections, the end of the dose distributions were found to coincide rather closely with the end of the activity distributions as seen in Figs. 6, 10, and 12. This is primarily due to the $1.2 \mathrm{~cm}$ resolution of the imaging system which is much larger than the $0.3 \mathrm{~cm}$ range of the treatment beam below the ${ }^{16} \mathrm{O}(p, n){ }^{15} \mathrm{O}$ reactions threshold energy (Table V). Since over $80 \%$ of the positron-emitting activity during and shortly after the treatment is due to the decay of ${ }^{15} \mathrm{O}$, this will be true for imaging systems with resolutions down to roughly $0.5 \mathrm{~cm}$.

Figures 9 and 10 show that there are strong correlations between the radioisotopic activities and the composition of the tissue. While the extent of the adipose deposit along the beam path was only one-third the resolution of the imaging system, the correlations in the ${ }^{15} \mathrm{O}$ and ${ }^{11} \mathrm{C}$ radioisotopic vectors were still significant. Such correlations would be stronger with more extended inhomogeneities or with an imaging system with better spatial resolution. While the dose deposited in various inhomogeneities may or may not vary signifi- 
TABLE V. The primary proton-induced reactions in tissue which produce positron-emitting isotopes.

\begin{tabular}{lccc}
\hline \hline \multicolumn{1}{c}{ Reaction } & $\begin{array}{c}\text { Threshold energy, } \\
\text { lab frame }(\mathrm{MeV})\end{array}$ & $\begin{array}{c}Q \text {-value } \\
(\mathrm{MeV})\end{array}$ & $\begin{array}{c}\text { Proton range in } \\
\text { tissue, below } \\
\text { threshold }(\mathrm{cm})\end{array}$ \\
\hline${ }^{16} \mathrm{O}(p, 3 p 4 n){ }^{10} \mathrm{C}$ & 39.1 & -36.8 & 1.5 \\
$\left.{ }^{12} \mathrm{C}(p, p n)\right)^{11} \mathrm{C}$ & 20.3 & -18.7 & 0.4 \\
${ }^{14} \mathrm{~N}(p, \alpha){ }^{11} \mathrm{C}$ & 3.1 & -2.9 & 0.02 \\
$\left.{ }^{16} \mathrm{O}(p, \alpha p n)\right)^{11} \mathrm{C}$ & 27.5 & -25.9 & 0.8 \\
$\left.{ }^{14} \mathrm{~N}(p, p n)\right)^{13} \mathrm{~N}$ & 11.3 & -10.6 & 0.2 \\
${ }^{16} \mathrm{O}(p, \alpha){ }^{13} \mathrm{~N}$ & 5.5 & -5.2 & 0.04 \\
$\left.{ }^{14} \mathrm{~N}(p, n)\right)^{14} \mathrm{O}$ & 6.6 & -5.9 & 0.06 \\
${ }^{16} \mathrm{O}(p, p 2 n){ }^{14} \mathrm{O}$ & 30.7 & -28.9 & 0.9 \\
$\left.{ }^{16} \mathrm{O}(p, p n)\right)^{15} \mathrm{O}$ & 16.6 & -15.7 & 0.3 \\
${ }^{18} \mathrm{O}(p, n){ }^{18} \mathrm{~F}$ & 2.6 & -2.4 & 0.01 \\
\hline \hline
\end{tabular}

cantly compared to the surrounding tissues, these radioisotopic correlations may be used as reference points for range verification relative to anatomic features along the beam path.

The correlations observed in the radioisotopic images of Figs. 9 and 10 are due to the oxygen content dropping from $75 \%$ by mass in tissue to $28 \%$ by mass in adipose tissue, and the carbon content increasing from $11 \%$ to $60 \%$, respectively. Equation (A1) shows that the activity, and consequently the number of decays in any time interval, at any given depth varies linearly with the number density of target nuclei. Consequently, if the percent change in the density of a particular target nuclei is greater than the uncertainty in the reconstructed radioisotopic images, the compositional variation should be observable. This condition is common in tissues such as muscle, adipose, and bone, allowing their presence and location along the beam path to be identified. This condition is also present for hypoxic versus nonhypoxic tissue but not to the extent that they may be differentiated based on composition alone. For further discussion on the detection of hypoxia see Ref. 35.

\section{CONCLUSIONS}

Based on the results of these experiments it is seen that on-line range verification may be performed after a single typical macro-beam pulse with an on-line monitoring system. Calculations show that over $80 \%$ of the activity created in such short time scales is ${ }^{15} \mathrm{O}$, produced by the ${ }^{16} \mathrm{O}(p, p n){ }^{15} \mathrm{O}$ reaction. The range of the proton beam below this reaction's $16.6 \mathrm{MeV}$ threshold energy is $0.3 \mathrm{~cm}$. Since the best practical resolution for this type of system is very similar to this residual beam range, the end of the dose distribution and the activity distribution will coincide to within the resolution of foreseeable PET imaging systems. The effects of energy straggling and the momentum spread of the treatment beam would only be observable by means of the activity distribution at the end of the range for proton beams with energies above $150 \mathrm{MeV}$ using an improved imaging system with a resolution of $2-3 \mathrm{~mm}$.

In general it is very difficult to infer the dose distribution from the induced activity distribution alone. The total dose may be monitored more accurately by monitoring the increase in the total number of $511 \mathrm{keV}$ gamma-ray pairs detected between macro-pulses. The number of counts increases roughly linearly for treatment times less than about 2 min, at least in treatment volumes with low blood flow rates.

Variations in the elemental composition along the beam path are readily observed in layers of distinct tissue types. Variations as small as about 5\% in a single element, averaged over a resolvable region, may be identified. The detectable size of these compositional variations will change based on the uncertainty in the reconstructed isotopic images, which is determined by the dose delivered and the imaging time. It may be possible to detect smaller variations if two isotopic image vectors exhibit strong positive or negative correlations in the same region. This phenomenon may be used to verify the range relative to various anatomic features along the beam path. It may also be particularly useful if used with high-energy, low-intensity beams in conjunction with a proton-range telescope ${ }^{45}$ for transverse patient alignment. In this situation, the beam would traverse the prescribed volume at higher energies where the PET isotope reaction cross sections vary slowly, resulting in more reliable compositional determination across and beyond the tumor volume.

Range-verification capability will become increasingly important for improving dose localization. While it may be possible to reposition the patient and deliver the prescribed dose distributions with an uncertainty of just a few millimeters, it has been shown that internal organs, such as the prostate, may move $1-3 \mathrm{~cm}$ between treatments. ${ }^{46}$ In such cases, the advantage of the high dose localization offered by protons may be compromised or even detrimental without proper alignment and range verification for the shifting anatomic features.

The prototype on-line PET isotope monitoring system used for the experiments presented here was designed within a limited budget with the intention of investigating the feasibility of using PET isotopes as a monitor for proton radiotherapy beams. It should by no means be considered an optimally designed system for clinical use but rather as an experimental system suitable for initial investigations. To be clinically useful the solid angle should be increased to decrease the imaging time and the resolution should be improved to the subcentimeter range. In addition, rectangular crystals should be used with no intercrystal shielding, especially if only one-dimensional decay distributions are being reconstructed. In this case, depth-of-interaction blurring is removed by the calibration procedure and the image reconstruction algorithm. However, blurring will occur if the treatment beam is more than a few centimeters wide. ${ }^{33}$ Further design considerations and suggested improvements for online PET isotope monitoring devices may be found in Ref. 33.

\section{ACKNOWLEDGMENTS}

This work was supported by Grants Nos. PHY 9512104 and PHY 9208468 from The National Science Foundation 
and by grants from the following groups within the University of Michigan: The Office of the Vice President for Research, The John Munn Foundation through The Comprehensive Cancer Center, The College of Literature, Science and Art, The Rackham School of Graduate Studies, and The Department of Physics. Additional acknowledgments are also due to the many students who made valuable contributions to this research while participating in the NSF's Research Experience for Undergraduates (NSF REU) program: Armin Martin, Mary-Alice Abbott, Chris Hazard (two summers), Mark Chawla, and Chris Search. We also thank the NSCL accelerator operations staff for their technical assistance in producing the suitable high-energy pulsed proton beam needed for this work.

\section{APPENDIX: PRODUCTION OF POSITRON- EMITTING NUCLEI}

\section{A. Nuclear reactions in tissue which produce positron- emitting isotopes}

A small percentage of the particles in a charged-particle radiotherapy beam will undergo nuclear reactions with elements in the tissue during treatment. The positron-emitting isotopes which are produced during proton radiotherapy are determined by the nuclear isotopic composition of the treated tissue. The elemental composition of the swine-tissue used for these experiments are shown in Table I, along with the composition of human muscle and adipose tissue. The most abundant elements in tissue from which positron-emitting isotopes may be produced are oxygen, carbon, and nitrogen. While positron-emitting isotopes may be produced from other elements, they are inconsequential due to their low number density.

The most important reactions in tissue which produce positron-emitting isotopes are shown in Table $\mathrm{V}$, along with their threshold energy, $E_{i}^{T}$, and their $Q$-value. Also shown is the residual range of the proton in tissue for proton energies less than the reaction threshold energies. While the production of ${ }^{10} \mathrm{C}$ and ${ }^{18} \mathrm{~F}$ are not significant processes, both radioisotopes were observed in our experiments and are included for completeness. Other reactions are discussed in Ref. 35 . The positron-emitting isotopes which may be produced by protons from the isotopes of oxygen, carbon, and nitrogen are shown in Table A1 along with their half-lives, $t_{1 / 2 i}$, their endpoint energies, and the maximum range of the emitted positrons in tissue. ${ }^{47}$

\section{B. Continuous beams}

A general treatment of the production and decay of positron-emitting isotopes by proton radiotherapy beams has been presented in Ref. 29. Here, a detailed formulation is presented for analysis of our specific experimental results. The most important factors in the production of the so-called PET isotopes are the number of target nuclei per cubic centimeter, $n_{i}(\mathbf{r})$, the particle flux in particles per second per square centimeter, $\Phi(\mathbf{r}, t)$, the beam energy, $E(\mathbf{r})$, at all points in the treatment volume, and the energy-dependent
TABLE AI. Characteristics of the positron-emitting isotopes produced by protons incident on isotopes of oxygen, carbon, and nitrogen.

\begin{tabular}{cccc}
\hline \hline Isotope & $\begin{array}{c}\text { Half-life } \\
(\mathrm{s})\end{array}$ & $\begin{array}{c}\beta^{+} \text {endpoint } \\
\text { energy }(\mathrm{MeV})\end{array}$ & $\begin{array}{c}\text { Maximum } \beta^{+} \\
\text {range }(\mathrm{cm})\end{array}$ \\
\hline${ }^{10} \mathrm{C}$ & 19.3 & 1.87 & 0.9 \\
${ }^{11} \mathrm{C}$ & 1218 & 0.96 & 0.4 \\
${ }^{13} \mathrm{~N}$ & 598.2 & 1.19 & 0.5 \\
${ }^{14} \mathrm{O}$ & 70.6 & 1.81 & 0.9 \\
${ }^{15} \mathrm{O}$ & 122.2 & 1.72 & 0.8 \\
${ }^{18} \mathrm{~F}$ & 6588 & 0.64 & 0.3 \\
\hline \hline
\end{tabular}

cross sections of the target nuclei for the various PET reaction processes, $\sigma_{i p}(E)$. The energy is expressed in units of $\mathrm{MeV}$ and the cross section is expressed in units of barns $\left(1 \mathrm{~b}=10^{-24} \mathrm{~cm}^{2}\right)$.

For a small volume in the treatment field, the time rate of change in the number, $N_{i}$, of positron-emitting nuclei of type, $i$, depends on the production rate, by a process, $p$, and the decay rate. This is given by

$$
\frac{d N_{i p}(\mathbf{r}, t)}{d t}=\sigma_{i p}(E(\mathbf{r})) \Phi(\mathbf{r}, t) n_{i}(\mathbf{r}) A d z-\lambda_{i} N_{i}(\mathbf{r}, t),
$$

where the decay constant of the isotope is $\lambda_{i}=\ln (2) / t_{1 / 2}$ and the cross sectional area of the beam is $A$. If the beam flux is constant over time, $\Phi(\mathbf{r}, t)=\Phi(\mathbf{r})$, with a beam-pulse duration of $T_{\text {on }}$ seconds, the resultant number of nuclei from the process is

$N_{i p}(\mathbf{r}, t)=\frac{\mathcal{A}_{i p}^{\max }(\mathbf{r}, t)}{\lambda_{i}}\left\{\begin{array}{l}\left(1-e^{-\lambda_{l} t}\right) \quad \text { if } t \leqslant T_{\text {on }} \\ \left(1-e^{-\lambda_{i} T_{\text {on }}}\right) e^{-\lambda_{i}\left(l-T_{\text {on }}\right)}\end{array}\right.$ if $t \geqslant T_{\text {on }}$,

where $\mathcal{A}_{i p}^{\max }(\mathbf{r}, t)=\sigma_{i p}(E(\mathbf{r})) \Phi(\mathbf{r}) n_{i}(\mathbf{r}) A d z$ is the maximum activity of isotope, $i$, that can be produced in a differential volume through the process, $p$. The activity, $\mathcal{A}_{i p}(\mathbf{r}, t)$, is then given by

$$
\begin{aligned}
\mathcal{A}_{i p}(\mathbf{r}, t) & =\lambda_{i} N_{i p}(\mathbf{r}, t) \\
& =\mathcal{A}_{i p}^{\max }(\mathbf{r}, t)\left\{\begin{array}{l}
\left(1-e^{-\lambda_{i} t}\right) \quad \text { if } t \leqslant T_{\text {on }} \\
\left(1-e^{-\lambda_{l} T_{\mathrm{on}}}\right) e^{-\lambda_{i}\left(t-T_{\text {on }}\right)} \text { if } t \geqslant T_{\text {on }},
\end{array}\right.
\end{aligned}
$$

where time is measured from the moment the beam is turned on. Equation (A3) may be used to find the distribution of activity, due to isotope, $i$, which was created through process, $p$, by a radiotherapy beam with a constant flux over a period of time, $T_{\text {on }}$.

\section{Macro-pulsed beams}

Many of the accelerators used for proton radiotherapy are synchrotrons which deliver macroscopic pulsed beams due to the ramp time of the magnets. (In this treatment we ignore the microscopic, submicrosecond pulse structure of the beam due to the rf system.) In these cases the equations above are only applicable for one macro-pulse of the beam. If the accelerator has a period, $T=T_{\text {on }}+T_{\text {off }}$, then the activity 
present at any time is the sum of the activity remaining from each of the previous pulses. Suppose there are $N$ pulses delivered during a treatment. The amount of activity created through a given process, $p$, for each pulse is the same as given in Eq. (A3), when $t=T_{\text {on }}$. Accounting for the decay of the activity created by each pulse and summing, the activity present after the last beam pulse is

$$
\begin{aligned}
& \mathcal{A}_{i p}(\mathbf{r}, t) \\
& =\mathcal{A}_{i p}^{\max }(\mathbf{r}, t)\left(1-e^{-\lambda_{i} T_{\mathrm{on}}}\right)\left[e^{-\lambda_{i} T(N-1)}\right)+e^{\left.-\lambda_{i} T(N-2)\right)} \\
& \left.+\cdots+e^{\left.-\lambda_{i} T(N-N)\right)}\right] \\
& =\mathcal{A}_{i p}^{\max }(\mathbf{r}, t)\left(1-e^{-\lambda_{i} T_{\mathrm{on}}}\right) \\
& \times\left\{\begin{array}{l}
\sum_{j=0}^{n-1} e^{-j \lambda_{l} T} \text { after } n \text { pulses } \\
{\left[\sum_{j=0}^{N-1} e^{-j \lambda_{i} T}\right] e^{-\lambda_{i}\left(t-T N+T_{\text {off }}\right)} \quad \text { if } t \geqslant\left(T N-T_{\text {off }}\right)}
\end{array}\right.
\end{aligned}
$$

In the simplest case, PET activity is created by a continuous beam in a homogenous material of uniform density through one of the reactions shown in Table V. Generally, however, the PET activity distribution produced will be the result of many of these reactions, several of which may produce the same radioisotope through different reaction processes. The final PET activity distribution produced during the treatment is then the sum of these activity distributions and is given by

$$
\mathcal{A}(\mathbf{r}, t)=\sum_{i} \sum_{p} \mathcal{A}_{i p}(\mathbf{r}, t)
$$

The maximum activity achievable with pulsed beams may be investigated for radioisotopes with half-lives comparable to or shorter than the irradiation time. In Eq. (A4), if the number of pulses, $n$, is very large the series converges so that

$$
\begin{aligned}
\mathcal{A}_{i p}(\mathbf{r}, t) & =\mathcal{A}_{i p}^{\max }(\mathbf{r}, t)\left(1-e^{-\lambda_{i} T_{\mathrm{on}}}\right) \lim _{n \rightarrow \infty} \sum_{j=0}^{n-1} e^{-j \lambda_{i} T} \\
& =\mathcal{A}_{i p}^{\max }(\mathbf{r}) \quad \text { for } t \gg T .
\end{aligned}
$$

Isotopes whose half-lives are short compared to the irradiation time will approach an equilibrium activity level as the decay rate approaches the production rate.

\section{The particle flux throughout the treatment volume}

The particle flux term, $\Phi(\mathbf{r}, E, t)$, in the equations above describes the particle flux, energy, and energy distribution at all points in the treatment volume. The model of the energy and flux distributions is similar to other models. ${ }^{29}$ To calculate the distribution of positron-emitting activity, the energy as a function of depth must be determined for the material of interest. This is accomplished by numerically integrating the inverse of the total stopping power ${ }^{48}$ as follows, where $E_{o}$ is the initial beam energy and $z$ is the depth at which the beam has energy $E$ :

$$
z=\int_{E}^{E_{o}}\left(\frac{d E}{d x}\right)_{T}^{-1} d E .
$$

In addition to knowing the range-energy relationship it is also necessary to know the range number-distance curve. Particle flux is primarily lost through nonelastic nuclear interactions. At $250 \mathrm{MeV}$ and $150 \mathrm{MeV}$ approximately $31 \%$ and $17 \%$, respectively, of the protons passing through tissue are removed from the beam by nuclear processes. ${ }^{48}$ By comparison, our calculations for $150 \mathrm{MeV}$ protons in tissue show that about $3.5 \%$ of the beam flux produces positron-emitting isotopes through the reactions shown in Table V. It has been shown $^{29}$ that if $P_{\text {abs }}(E)$ is the probability that a particle is absorbed and undergoes a nuclear reaction before the end of its range, the fraction of the initial flux, $r_{\mathrm{abs}}^{E_{o}}(z)$, remaining at a given depth is

$$
r_{\mathrm{abs}}^{E_{o}}(z)=\frac{\left[1-P_{\mathrm{abs}}\left(E_{o}\right)\right]}{\left[1-P_{\mathrm{abs}}(E(z))\right]},
$$

where $E(z)$ is the beam energy as a function of depth. The resulting proton flux drops off with increasing depth as

$$
\Phi(z)=\Phi_{o} r_{\mathrm{abs}}^{E_{o}}(z),
$$

where $\Phi_{o}$ is the initial proton flux.

\section{E. Cross sections for producing positron-emitting isotopes}

Cross sections for the reactions listed in Table $\mathrm{V}$ are presented in Figs. 2(a) and 2(b). ${ }^{32,39,40,48-53}$ Due to the high number density of oxygen in tissue and the relatively high cross section for the ${ }^{16} \mathrm{O}(p, p n){ }^{15} \mathrm{O}$ reaction, ${ }^{15} \mathrm{O}$ is the most active isotope at the end of a typical treatment. Despite the relatively low number density of ${ }^{12} \mathrm{C}$ in tissue, a significant amount of ${ }^{11} \mathrm{C}$ activity is also produced by the $(p, p n)$ and $(p, p n \alpha)$ reactions due to the high production cross section on ${ }^{12} \mathrm{C}$ and the abundance of ${ }^{16} \mathrm{O}$. As noted earlier, the threshold energies, as measured in the reference frame of the lab, vary from 2.6 to $39.1 \mathrm{MeV}$, below which the production goes to zero. The endpoint of the proton's range is therefore slightly beyond the distal end of the activity distribution as given in Table V. Consequently, the end of the activity distribution and the end of the proton's range will not occur at the same depth. It is shown in Table II that over $80 \%$ of the positron-emitting activity imaged on short time scales is due to the decay of ${ }^{15} \mathrm{O}$. The range of the treatment beam below the reaction threshold for the ${ }^{16} \mathrm{O}(p, p n){ }^{15} \mathrm{O}$ reaction is only $0.3 \mathrm{~cm}$. Thus for on-line imaging, the end of the ${ }^{15} \mathrm{O}$ activity distribution provides the best marker for the range of the treatment beam.

\section{F. Correlation between total dose and total activity}

The possibility of discerning the dose distribution from the nuclear decay distribution of long-lived positron-emitting activity created by the treatment beam has previously been explored by other investigators. ${ }^{27,29}$ Because the physical mechanisms by which nuclear and atomic interactions occur 
are not related, it is difficult to extract the dose distribution from the nuclear decay distribution. This has been demonstrated with ${ }^{11} \mathrm{C}$ decay distributions produced by proton beams in Lucite. ${ }^{29}$ It is possible, however, to monitor the integrated dose delivered by monitoring the total number of annihilation events detected as a function of time. The total dose delivered after $n$ pulses is given by

$$
D=\frac{\text { energy }}{\text { mass }}=\frac{P_{\text {beam }} t}{\rho_{T} V}=\frac{E_{o} \Phi_{o}\left(n T_{\text {on }}\right)}{\rho_{T} V},
$$

where $P_{\text {beam }}=E_{o} \Phi_{o}$ is the power delivered by the beam. The units of the dose are in Gray if the beam energy is in $\mathrm{MeV}$, the beam current, $\Phi_{o}$, is in microamps, $T_{\text {on }}$ is in seconds, and the mass of the treated volume is in kilograms. The total activity present after $n$ pulses is given by integrating Eq. (A5) over the treatment volume. After performing the integral and solving for the initial beam current, the total dose after $n$ pulses, $D(n)$, is found to be related to the activity by the relationship

$$
D(n)=\frac{E_{o} n}{\rho_{T} V} \frac{N_{n}}{\sum_{i} \Sigma_{p}\left[Y_{i p} \sum_{j=0}^{n-1} e^{-j \lambda_{i} T}\right]} .
$$

Here, $N_{n}=\mathcal{A}(t) T_{\text {on }}=\mathcal{A}(t) T_{\text {off }}$ is the number of decays between beam pulses $n$ and $(n+1)$, and

$$
Y_{i p}=\left(1-e^{-\lambda_{i} T_{\mathrm{on}}}\right) \int_{0}^{R} \sigma_{i p}(E(z)) r_{\mathrm{abs}}^{E_{o}}(z) n_{i}(z) A d z
$$

is the yield of radioisotope, $i$, by the process, $p$, per (proton $/ \mathrm{cm}^{2}$ ) per pulse.

The number of decays between successive beam pulses will eventually become constant if the treatment time is long compared to the half-life of the most significant, longestlived isotope, which is typically ${ }^{11} \mathrm{C}$. However, if the treatment time is less than or comparable to the half-life of the shortest-lived isotope, the number of decays detected between each beam pulse will be approximately linear with dose. Clinical treatment times are typically less than 2 min, or less than one half-life of ${ }^{15} \mathrm{O}$ which is the shortest-lived isotope produced in abundance. In Fig. 11 it is shown that on short time scales the number of decay events detected between beam pulses increases roughly linearly with the treatment time for macro-pulsed beams. Consequently, it is possible, within certain limitations, to monitor the integrated dose delivered by monitoring the total number of annihilation events detected between beam pulses.

\section{G. Activity and dose calculations}

A simulation code was written to estimate the depthdistribution of PET isotopes produced by proton beams in materials of varying composition. Several layers of materials of differing composition may be placed in series. The stopping power of the material is

$$
\frac{1}{\rho_{T}}\left(\frac{d E}{d z}\right)_{T}=\sum_{i} \frac{1}{\rho_{i}}\left(\frac{d E}{d z}\right)_{i},
$$

where $(d E / d z)_{i}$ is the stopping power of the individual elements. Stopping power values are obtained from spline fits to the values given in relevant data compilations. ${ }^{48}$ The dose and energy as a function of depth are then found by numerically integrating Eq. (A13). Once the energy as a function of depth, $E(z)$, is known the energy-dependent PET-isotope production cross sections may be mapped into depthdependent cross sections. The depth-dependent particle flux is calculated from Eq. (A8). Range straggling and scattering have not been implemented in the code. The isotopic distribution of PET activity is then calculated in $0.2 \mathrm{~cm}$ increments with Eq. (A5) using Eq. (A3) for continuous beams and Eq. (A4) for pulsed beams. The total and isotopic activity distributions may be integrated over the experimental imaging time and compared directly with experimental results.

${ }^{a)}$ Author for correspondence: Dale Litzenberg, The University of Michigan Medical Center, Dept. of Rediation Oncology, UH-Rm. B2C432B Box 0010, 1500 E. Medical Center Dr., Ann Arbor, MI 48109, phone: (734) 763-9836, FAX: (734) 936-7859, electronic mail: litzen@umich.edu

${ }^{1}$ P. L. Petti, "'Differential-pencil-beam dose calculations for charged particles," Med. Phys. 19, 137-149 (1992).

${ }^{2} \mathrm{M}$. Goitein, "The measurement of tissue heterodensity to guide charged particle radiotherapy," Int. J. Radiat. Oncol., Biol., Phys. 3, 27-33 (1977).

${ }^{3}$ M. Goitein and J. M. Sisterson, "The influence of thick inhomogeneities on charged particle beams,' Radiat. Res. 74, 217-230 (1978).

${ }^{4} \mathrm{M}$. Goitein, "Compensation for inhomogeneities in charged particle radiotherapy using computed tomography,' Int. J. Oncol., Biol., Phys. 4, 499-508 (1978).

${ }^{5}$ A. A. Mustafa and D. F. Jackson, "The relation between X-ray CT numbers and charged particle stopping powers and its significance for radiotherapy treatment planning," Phys. Med. Biol. 28, 169-176 (1983).

${ }^{6}$ E. L. Alpen, W. Saunders, A. Chatterjee, J. Llacer, G. T. Y. Chen, and J. Scherer, "A comparison of water equivalent thickness measurements: CT method vs heavy ion beam technique," Br. J. Radiol. 58, 542-548 (1985).

${ }^{7}$ C. A. Tobias, "Analysis of microcomposition of biological tissue by means of induced radioactivity," Science 109, 109-113 (1949).

${ }^{8}$ M. V. Mayneord, J. H. Martin, and D. A. Layne, "Production of radioactivity in animal tissues by high-energy X-rays," Nature (London) 164, 728-730 (1949).

${ }^{9}$ W. L. Hughes, G. H. Nussbaum, R. Connolly, B. Emami, and P. Reilly, "Tissue perfusion rate determined from the decay of oxygen-15 activity after photon activation in situ," Science 204, 1215-1216 (1979).

${ }^{10}$ R. K. Ten Haken, G. H. Nussbaum, B. Emami, and W. L. Hughes, "Photon activation- ${ }^{15} \mathrm{O}$ decay studies of tumor blood flow," Med. Phys. 8, 324-336 (1981).

${ }^{11} \mathrm{~S}$. Graffman and B. Jung, ${ }^{\prime 11} \mathrm{C}$ and ${ }^{15} \mathrm{O}$ induced in the mouse by 175 MeV protons,"' Acta Radiol.: Ther., Phys., Biol. 14, 113-126 (1975).

${ }^{12}$ G. W. Bennett, J. O. Archambeau, B. E. Archambeau, J. I. Meltzer, and C. L. Wingate, "Visualization and transport of positron emission from proton activation in vivo," Science 200, 1151-1152 (1978).

${ }^{13}$ R. K. Ten Haken, M. Awschalom, and I. Rosenberg, "Activation of the major constituents of tissue and air by a fast neutron radiation therapy beam,"' Med. Phys. 10, 636-641 (1983).

${ }^{14}$ M. C. Taylor, "Pion cancer therapy: Positron activity as an indicator of depth-dose,' Science 169, 377-378 (1970).

${ }^{15}$ L. F. Mausner, M. A. Moinester, J. D. Knight, S. Cochavi, and S. S. Friedland, "in vivo beam localization by positron activation in pion therapy,' Radiat. Res. 80, 10-23 (1979).

${ }^{16}$ H. Shirato, R. Harrison, R. O. Kornelsen, G. K. Y. Lam, C. C. Gaffney, G. B. Goodman, E. Grochowski, and B. Pate, "Detection of pion-induced radioactivity by autoradiography and positron emission tomography," Med. Phys. 16, 388-345 (1989).

${ }^{17}$ H. D. Maccabee, U. Madhvanath, and M. R. Raju, "Tissue activation studies with alpha-particle beams," Phys. Med. Biol. 14, 213-224 (1969).

${ }^{18}$ J. Llacer, J. B. Schmidt, and C. A. Tobias, "Characterization of frag- 
mented heavy-ion beams using a three-stage telescope detector: Measurements of 670-MeV/amu ${ }^{20} \mathrm{Ne}$ beams," Med. Phys. 17, 151-157 (1990).

${ }^{19}$ W. Enghardt, W. D. Fromm, H. Geissel, H. Heller, G. Kraft, A. Magel, P. Manfrass, G. Munzenberg, F. Nickel, J. Pawelke, D. Schardt, C. Scheidenberger, and Sobiella, "The spatial distribution of positron-emitting nuclei generated by relativistic light ion beams in organic matter,' Phys. Med. Biol. 37, 2127-2131 (1992).

${ }^{20}$ S. M. Seltzer, "An assessment of the role of charged secondaries from nonelastic nuclear interactions by therapy proton beams in water," $\mathrm{Na}-$ tional Institute of Standards and Technology report NISTIR 5221, June, 1993.

${ }^{21}$ R. F. Laitano, M. Rosetti and M. Frisoni, "Effects of nuclear interactions on energy and stopping power in proton beam dosimetry," Nucl. Instrum. Methods Phys. Res. A 376, 446-476 (1996).

${ }^{22}$ G. W. Bennett, A. C. Goldberg, G. S. Levine, J. Guthy, J. Balsamo, and J. O. Archambeau, "Beam localization via ${ }^{15} \mathrm{O}$ activation in protonradiation therapy," Nucl. Instrum. Methods 125, 333-338 (1975).

${ }^{23} \mathrm{R}$. Loevinger and M. Berman, "A formalism for calculation of absorbed dose from radionuclides," Phys. Med. Biol. 13, 205-217 (1976).

${ }^{24}$ J. Llacer, A. Chatterjee, E. L. Alpen, W. Saunders, S. Andreae, and H. C. Jackson, "Imaging by injection of accelerated radioactive particle beams," IEEE Trans. Med. Imaging 3, 80-90 (1984).

${ }^{25}$ D. W. Litzenberg, J. F. Bajema, F. D. Becchetti, J. A. Brown, R. S. Raymond, D. A. Roberts, J. Caraher, G. Hutchins, R. Ronningen, R. Smith, and M. Abbott, "On-line monitoring and PET imaging of proton radiotherapy beams," IEEE Med. Imag. Conf. Rec., pp. 954-956, Orlando, FL, 25-31, Oct. 1992.

${ }^{26}$ D. W. Litzenberg, J. F. Bajema, F. D. Becchetti, D. A. Roberts, R. Ronningen, A. M. Vander Molen, J. A. Brown, R. K. Ten-Haken, J. Caraher, C. Hazard, and M. Chawla, "On-line monitoring and P.E.T. imaging of proton radiotherapy beams," IEEE Med. Imag. Conf. Rec. pp. 16721676, Norfolk, VA, Oct. 30-Nov. 5, 1994.

${ }^{27}$ S. Vynckier, S. Derreumaux, F. Richard, A. Bol, C. Michel, and A. Wambersie, "Is it possible to verify directly a proton-treatment plan using positron emission tomography," Radiother. Oncol. 26, 275-277 (1993).

${ }^{28}$ A. M. J. Paans and J. M. Schippers, "Proton therapy in combination with PET as monitor: A feasibility study," IEEE Trans. Nucl. Sci. 40, 10411044 (1993).

${ }^{29}$ U. Oelfke, G. K. Y. Lam, and M. S. Atkins, "Proton dose monitoring with PET: Quantitative studies in Lucite,’ Phys. Med. Biol. 41, 177-196 (1996).

${ }^{30}$ J. Pawelke, L. Byars, W. Enghardt, W. D. Fromm, H. Geissel, B. G. Hasch, K. Lauckner, P. Manfrass, D. Schardt, and M. Sobiella, "The investigation of different cameras for in-beam PET imaging," Phys. Med. Biol. 41, 279-296 (1996).

${ }^{31}$ A. Del Guerra and G. Di Domenico, "Positron Emission Tomography as an aid to in vivo dosimetry for proton radiotherapy: A Monte Carlo simulation," TERA 93/10 TRA 9, Aug. 1993.

${ }^{32}$ M. G. Albouy, J. P. Cohen, M. Gusakow, N. Poffe, H. Sergolle, and L. Valentine, "Spallation de l'oxygene par besprotons de 20 a $150 \mathrm{MeV}$," Phys. Lett. 2, 306-307 (1962).

${ }^{33}$ D. W. Litzenberg, F. D. Becchetti, and D. A. Roberts, "On-line PET monitoring of radiotherapy beams: Image reconstruction and monte carlo simulations of detector geometries," IEEE Trans. Nucl. Sci. 44, 16461657 (1997)

${ }^{34}$ D. W. Litzenberg, F. D. Becchetti, D. A. Roberts, A. M. Vander Molen, and Ron Fox, "A parallel, transputer-based data acquisition system for on-line PET imaging of proton and gamma-ray radiotherapy beams," IEEE Trans. Nucl. Sci. 43, 154-158 (1996).
${ }^{35} \mathrm{D}$. W. Litzenberg, "'On-line monitoring and PET imaging of the positronemitting activity created in tissue by proton radiotherapy beams," Ph.D. dissertation, University of Michigan (1997).

${ }^{36} \mathrm{H}$. Stark and J. W. Woods, Probability, Random Processes and Estimation Theory for Engineers, 2nd ed. (Prentice Hall, New Jersey, 1994), p. 251.

${ }^{37}$ P. R. Bevington and D. K. Robinson, Data Reduction and Error Analysis for the Physical Sciences, 2nd ed. (McGraw-Hill, New York, 1992), pp. 194-219.

${ }^{38}$ ICRU, “Tissue substitutes in radiation dosimetry and measurement," Report 44 of the International Commission on Radiation Units and Measurements (Bethesda, MD, 1989).

${ }^{39}$ S. W. Kitwanga, P. Leleux, P. Lipnik, and J. Vanhorenbeeck, "Production of ${ }^{14} \mathrm{O},{ }^{15} \mathrm{O},{ }^{18} \mathrm{~F}$ and ${ }^{19} \mathrm{Ne}$ radioactive nuclei from $(p, n)$ reactions up to $30 \mathrm{MeV}$," Phys. Rev. C 42, 748-752 (1990).

${ }^{40} \mathrm{M}$. B. Chadwick and P. G. Young, "Proton nuclear interactions up to 250 MeV for radiation transport simulations of particle therapy," Proceedings of the PTCOG Conference to be published in Journal of Endocurietherapy/Hyperthermia Oncology, Detroit, 1996.

${ }^{41}$ P. G. Young, E. D. Arthur and M. B. Chadwick, "Comprehensive nuclear model calculations: Introduction to the theory and use of the GNASH code," Los Alamos National Laboratory document LA-12343-MS (1992).

${ }^{42} \mathrm{R}$. Eilbert, "Bone calcium determination in vivo by proton activation analysis," Ph. D. dissertation, Harvard University (1975).

${ }^{43}$ M. B. Chadwick and P. G. Young, "Calculation and evaluation of cross sections and kerma factors for neutrons up to $100 \mathrm{MeV}$ on ${ }^{16} \mathrm{O}$ and ${ }^{14} \mathrm{~N}$,", Nucl. Sci. Eng. 123, 1-16 (1996).

${ }^{44}$ M. B. Chadwick and P. G. Young, "Calculation and evaluation of cross sections and kerma factors for neutrons up to $100 \mathrm{MeV}$ on carbon," Nucl. Sci. Eng. 123, 17-37 (1996).

${ }^{45}$ M. J. Inwood, S. Thomson, and N. J. Bryant, "Hematology," Philadelphia: in Lynch's Medical Laboratory Technology, 4th ed. edited by S. S. Raphael (W. B. Saunders, 1983), pp. 625-627.

${ }^{46}$ J. L. Romero, J. H. Osborne, F. P. Brady, W. Caskey, D. A. Cebra, M. D. Partlan, B. H. Kusko, R. S. King, I. Mirshad, H. Kubo, I. Daftari, and W. $\mathrm{Chu}$, "Patient positioning for protontherapy using a proton range telescope," Nucl. Instrum. Methods Phys. Res. A 356, 558-565 (1995).

${ }^{47}$ J. M. Balter, K. L. Lam, H. M. Sandler, J. F. Littles, R. L. Bree, and R. K. Ten Haken, "Automated localization of the prostate at the time of treatment using implanted radiopaque markers: Technical feasibility," Int. J. Radiat. Oncol., Biol., Phys. 33, 1281-1286 (1995).

${ }^{48}$ M. J. Berger and S. M. Seltzer, "Tables of energy loss and ranges of electrons and positrons," in Studies in Penetration of Charged Particles in Matter, edited by U. Fano, Publ. No. 1133 (National Academy of Sciences, National Research Council, Washington D.C., 1956), pp. 1-30.

${ }^{49}$ J. F. Janni, “Proton range-energy tables, $1 \mathrm{keV}-10 \mathrm{GeV}$,' At. Data Nucl. Data Tables 27, (1982).

${ }^{50} \mathrm{D}$. F. Measday, "The ${ }^{12} \mathrm{C}(p, p n){ }^{11} \mathrm{C}$ reaction from 50 to $160 \mathrm{MeV}$," Nucl. Phys. 78, 476-480 (1966).

${ }^{51} \mathrm{M}$. Epherre and C. Seide, "Excitation functions of ${ }^{7} \mathrm{Be}$ and ${ }^{11} \mathrm{C}$ produced in nitrogen by low-energy protons," Phys. Rev. C 3, 2167-2171 (1971).

${ }^{52}$ L. Valentin, G. Albouy, J. P. Cohen, and M. Gusakow, "Fonctions d'excitation des reactions $(p, p n)$ et $(p, 2 p 2 n)$ dans les noyaux legers entre 15 et $155 \mathrm{MeV}$," J. Phys. (France) 25, 704-706 (1964).

${ }^{53}$ A. B. Clegg, "Gamma radiation from medium energy proton bombardment of lithium, beryllium, boron, carbon and nitrogen,' Proc. Phys. Soc. 78, 681 (1961). 